\title{
Enantioselective Construction of Quaternary Stereogenic Carbons by the Lewis Base Catalyzed Additions of Silyl Ketene Imines to Aldehydes
}

\author{
Scott E. Denmark, * Tyler W. Wilson, Matthew T. Burk, John R. Heemstra, Jr. \\ Department of Chemistry, University of Illinois Urbana-Champaign, Urbana, Illinois 61801
}

\section{SUPPORTING INFORMATION}

Table of Contents

Page

General Experimental

Literature Preparations

Experimental Procedures

Preparation of Silyl Ketene Imines

Addition of Silyl Ketene Imines to Benzaldehyde

Addition of Different Silyl Ketene Imines to 2-Naphthaldehyde

Addition of Silyl Ketene Imine 3a to Different Aldehydes

References

${ }^{1} \mathrm{H}$ NMR Spectra

\section{General Experimental}

All reactions were performed in oven dried $\left(140^{\circ} \mathrm{C}\right)$ or flame dried glassware under an atmosphere of dry argon. Tetrahydrofuran (Fisher, HPLC grade) and $\mathrm{CH}_{2} \mathrm{Cl}_{2}$ (Fisher, HPLC grade) were dried by percolation through neutral alumina in a solvent dispensing system. ACS reagent grade diethyl ether, ethyl acetate, and hexanes used for chromatography and recrystallization were purchased from Fisher. Dichloromethane, benzene, and diisopropyl ether for chromatography and recrystallization were ACS reagent grade. Column chromatography was performed using Silicycle Flash-P 40-60 $\mathrm{m}$ silica gel. Silicon tetrachloride was heated at reflux for $24 \mathrm{~h}$ and was distilled prior to use. Diisopropylamine was freshly distilled over $\mathrm{CaH}_{2}$. Benzaldehyde (4a), 4-methoxybenzaldehyde (4f), 2-tolualdehyde $\quad(\mathbf{4 g}), \quad$ 4trifluoromethylbenzaldehyde (4c), 1-naphthaldehyde (4h) and 2-furaldehyde (4i) were distilled under reduced pressure before use. Methyl 4-formylbenzoate (4e), 4-bromobenzaldehyde (4d), and 2-naphthaldehyde (4b) were sublimed under high vacuum and stored in a glove box. 3,5- 
Dinitrobenzoyl chloride was triturated with petroleum ether. Methyl iodide, ethyl iodide, isobutyl bromide, iso-propyl bromide, allyl bromide, benzyl cyanide, cyclohexaneanecarbonitirle, 4-methoxybenzyl cyanide, 2-methylbenzonitrile, 4-trifluoromethylbenzyl cyanide, $t$ butyldimethylchlorosilane were used as received. $n$-Butyllithium was titrated prior to use. ${ }^{1}$ Internal temperature of low temperature reactions was measured using Teflon coated thermocouples. $(R, R)-\mathbf{5}$ catalyst was obtained from ObiterResearch, LLC.

${ }^{1} \mathrm{H} \mathrm{NMR}$ and ${ }^{13} \mathrm{C} \mathrm{NMR}$ spectra were acquired in $\mathrm{CDCl}_{3}$ at $500 \mathrm{MHz}$ and referenced to residual $\mathrm{CHCl}_{3}$ at 7.26 and 77.00 ppm respectively or in $\mathrm{C}_{6} \mathrm{D}_{6}$ and referenced to residual $\mathrm{C}_{6} \mathrm{D}_{5} \mathrm{H}$ at 7.15 and $128.00 \mathrm{ppm}$ respectively. Assignments were obtained by reference to COSY, HMQC and HMBC correlations. Chemical shifts are reported in ppm, multiplicities are indicated by $\mathrm{s}$ (singlet), d (doublet), t (triplet), q (quartet), p (pentet), h (hextet), hep (heptet), m (multiplet) and br (broad). Coupling constants, $J$, are reported in Hertz. Mass spectrometry was performed by the University of Illinois Mass Spectrometer Center. EI mass spectra were performed on a 70VSE instrument. ESI mass spectra were performed on a Waters Q-Tof Ultima instrument. Data are reported in the form of $(\mathrm{M} / \mathrm{Z})$ versus intensity. Infrared spectra (IR) were recorded in $\mathrm{KBr}$ pellets. Peaks are reported in $\mathrm{cm}^{-1}$ with indicated relative intensities: s (strong, 67-100\%); $\mathrm{m}$ (medium, 34-66\%); w (weak, 0-33\%). Melting points (mp) were determined in sealed tubes and are corrected. Analytical supercritical fluid chromatography was performed on a Berger Instruments SFC with spectrophotometric detector $(220 \mathrm{~nm})$ using Daicel Chiralpak OD, OJ, $\mathrm{OB}, \mathrm{AD}$ and AS columns as well as a Regis Whelk-O1 column. Optical rotations were measured in Fischer ACS reagent grade $\mathrm{CHCl}_{3}$ and are reported as follows: concentration $(\mathrm{c}=\mathrm{g} / \mathrm{dL})$, and solvent.

\section{Literature Preparations}

2-Phenylpropanenitrile, ${ }^{2}$ 2-phenylbutanenitrile, ${ }^{3}$ 2-phenyl-pent-4-enenitrile, ${ }^{2} 3$-methyl-2phenyl-butanenitrile, $^{3} \quad$ 4-methyl-2-phenylpentanenitrile, ${ }^{3} \quad$ 2,3-dimethyl-butanenitrile, ${ }^{4} \quad$ 2-(4methoxyphenyl)-propanenitrile, ${ }^{3} \quad$ 2-(2-methylphenyl)propanenitrile, ${ }^{3} \quad$ 2-phenyl 2-(4(trifluoromethyl)phenyl)-butanenitrile, ${ }^{5}$ were prepared according to the general procedure of Watt except diisopropylamine was used in place of $N$-isopropylcyclohexaneylamine. ${ }^{2} 1$-(1,1dimethylethyl)-1,1-dimethyl- $N$-(2-phenyl-1-propen-1-ylidene)silanamine $\quad(3 \mathbf{3 a}),{ }^{4} \quad 1-(1,1-$ dimethylethyl)-1,1-dimethyl- $N$-(2-phenyl-1-buten-1-ylidene)silanamine $\quad(\mathbf{3 e}){ }^{4} \quad 1-(1,1-$ 
dimethylethyl)-1,1-dimethyl- $N$-(4-methyl-2-phenyl-1-pentenylidene)silanamine $\quad(\mathbf{3 f}){ }^{4} \quad 1-(1,1-$ dimethylethyl)-1,1-dimethyl- $N$-(2-phenyl-1,4-pentadien-1-ylidene)silanamine $\quad(\mathbf{3 h}){ }^{6} \quad \mathrm{~N}$ (cyclohexaneylidenemethylene)-1-(1,1-dimethylethyl)-1,1-dimethylsilanamine $\quad(3 \mathbf{3})^{6} \quad$ were prepared according to the general procedure of $\mathrm{Fu}$ and coworkers except cannula filtration was substituted for filtration in a glove box. ${ }^{4}$ 1-(1,1-Dimethylethyl)-1,1-dimethyl- $N$-[2-[4(trifluoromethyl)phenyl]-1-butenylidene]silanamine $(\mathbf{3 b})^{4}$ was prepared according to the modified procedure of $\mathrm{Fu}$ and co-workers except cannula filtration was substituted for filtration in a glove box, and the reaction was run at $-98^{\circ} \mathrm{C}$ in a $\mathrm{MeOH} / \mathrm{N}_{2}(1)$ bath. ${ }^{4}$

\section{General Procedure 1. Preparation of Silyl Ketene Imines.}

Preparation of 1-(1,1-Dimethylethyl)-1,1-dimethyl- $N$-(3-methyl-2-phenyl-1-buten-1ylidene)silanamine (3g)
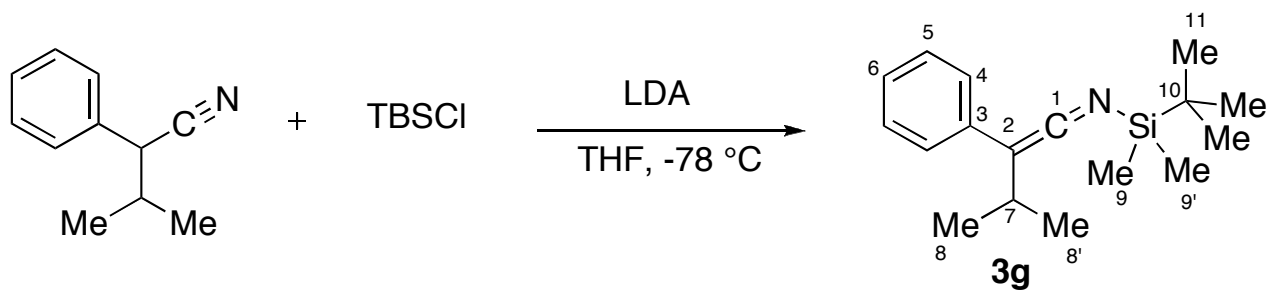

$n$-Butyllithium (2.6 $\mathrm{M}$ in hexanes, $2.26 \mathrm{~mL}, 5.98 \mathrm{mmol}, 1.0$ equiv) was added via syringe to a flame-dried, $50-\mathrm{mL}$, Schlenk flask containing a $-78^{\circ} \mathrm{C}$ (dry ice/acetone bath, internal temp) solution of $0.60 \mathrm{~g}$ diisopropylamine $(5.98 \mathrm{mmol}, 1.0$ equiv) in $6 \mathrm{~mL}$ of THF. The reaction mixture was stirred for $10 \mathrm{~min}$ at $-78{ }^{\circ} \mathrm{C}$ and then a solution of $0.95 \mathrm{~g}$ (5.98 mmol) 3-methyl-2phenylbutanenitrile in $4 \mathrm{~mL}$ of THF was added to the reaction via cannula. The resultant yellow solution was stirred for $5 \mathrm{~min}$ at $-78{ }^{\circ} \mathrm{C}$ and then a solution of $0.99 \mathrm{~g}$ of $\mathrm{TBSCl}$ (6.59 $\mathrm{mmol}, 1.1$ equiv) in $4 \mathrm{~mL}$ of THF was added via cannula. The solution was allowed to warm to room temperature and was stirred for $1 \mathrm{~h}$. The solvent was removed under high vacuum $(0.3 \mathrm{~mm} \mathrm{Hg})$, and the residue taken up in $30 \mathrm{~mL}$ of anhydrous pentane and was then filtered via cannula into a flame dried, $100-\mathrm{mL}$, round-bottomed flask. Removal of the solvent under vacuum $(0.3 \mathrm{mmHg})$ afforded $1.59 \mathrm{~g}(97 \%)$ of $\mathbf{3 g}$ as a yellow liquid, which was used in the subsequent addition reactions without further purification. 


\section{Data for 3g:}

${ }^{1} \mathrm{H}$ NMR: $\quad\left(500 \mathrm{MHz}, \mathrm{C}_{6} \mathrm{D}_{6}\right)$

7.27-7.22 (m, $4 \mathrm{H}, \mathrm{HC}(4)$ and $\mathrm{HC}(5)), 6.95-6.91(\mathrm{~m}, 1 \mathrm{H}, \mathrm{HC}(6)), 2.69$ (qq, $J=6.7$,

6.7, $1 \mathrm{H}, \mathrm{HC}(7)), 1.20\left(\mathrm{~d}, J=6.8,6 \mathrm{H}, \mathrm{H}_{3} \mathrm{C}(8)\right.$ and $\left.\mathrm{H}_{3} \mathrm{C}\left(8^{\prime}\right)\right), 0.92$ (s, $6 \mathrm{H}^{\prime} \mathrm{H}_{3} \mathrm{C}(9)$ and $\mathrm{H}_{3} \mathrm{C}\left(9^{\prime}\right), 0.10$ (s, 9H, $\left.\mathrm{H}_{3} \mathrm{C}(11)\right)$

${ }^{13} \mathrm{C}$ NMR: $\quad\left(125 \mathrm{MHz}, \mathrm{C}_{6} \mathrm{D}_{6}\right)$

$186.0(\mathrm{C}(1)), 138.3(\mathrm{C}(4)), 129.0(\mathrm{C}(5)), 123.8(\mathrm{C}(6)), 122.6(\mathrm{C}(3)), 62.3(\mathrm{C}(2))$, $25.6(\mathrm{C}(11)), 24.8(\mathrm{C}(7)), 22.8\left(\mathrm{C}\left(8,8^{\prime}\right)\right), 17.7(\mathrm{C}(10)),-4.8\left(\mathrm{C}\left(9,9^{\prime}\right)\right)$

IR: (neat)

3022 (w), 2955 (s), 2929 (s), 2859 (m), 2030 (s), 1595 (s), 1495 (s), 1463 (m), 1251

(s), $1071(\mathrm{~m}), 976(\mathrm{~m}), 840(\mathrm{~s}), 822$ (s), $784(\mathrm{~s}), 752$ (s), $732(\mathrm{~m}), 692(\mathrm{~m})$

MS: $(\mathrm{EI}, 70 \mathrm{eV})$

73 (77), 135 (20), 161 (18), 207 (16), 275 (100)

HRMS: Calcd for $\mathrm{C}_{17} \mathrm{H}_{27} \mathrm{NSi}$ : 273.1913, found: 273.1913

\section{Preparation of 1-(1,1-Dimethylethyl)-1,1-dimethyl- $N$-(3-methyl-2-methyl-1-buten-1-} ylidene)silanamine $(3 \mathbf{j})$
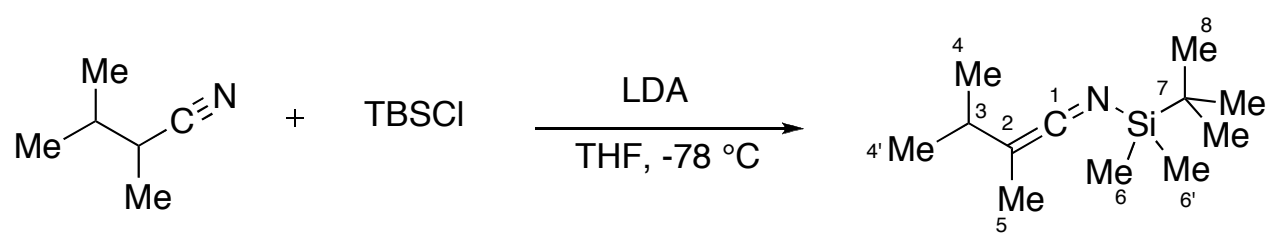

3j

Following General Procedure 1, $i-\mathrm{Pr}_{2} \mathrm{NH}$ (2.90 mL, $20.6 \mathrm{mmol}, 1.0$ equiv), THF (15 mL), 2.6 M $n$-BuLi in hexanes (7.9 mL 1.0 equiv), 2,3-dimethylbutanenitrile (2.06 g, $20.6 \mathrm{mmol})$ in 8 $\mathrm{mL}$ of THF and a solution of TBSCl (3.42 g, 1.1 equiv, $20.6 \mathrm{mmol})$ in $8 \mathrm{~mL}$ of THF were combined to afford $3.27 \mathrm{~g}(75 \%)$ of $\mathbf{3 j}$ after vacuum distillation at $3 \mathrm{mmHg}^{7}$

Data for 3j:

bp: $\quad 62-63^{\circ} \mathrm{C}(3 \mathrm{mmHg})$

${ }^{1} \mathrm{H}$ NMR: $\quad\left(500 \mathrm{MHz}, \mathrm{C}_{6} \mathrm{D}_{6}\right)$

2.08 (qq, $J=6.6,6.6,1 \mathrm{H}, \mathrm{HC}(3)), 1.068\left(\mathrm{~d}, J=6.6 \mathrm{~Hz}, 6 \mathrm{H}, \mathrm{H}_{3} \mathrm{C}(4)\right.$ and $\left.\mathrm{H}_{3} \mathrm{C}\left(4^{\prime}\right)\right), 0.96$ (s, $\left.9 \mathrm{H}, \mathrm{H}_{3} \mathrm{C}(8)\right),-0.12$ (s, $6 \mathrm{H}, \mathrm{H}_{3} \mathrm{C}(6)$ and $\mathrm{H}_{3} \mathrm{C}\left(6^{\prime}\right)$ ) 
${ }^{13} \mathrm{C} \mathrm{NMR:} \quad\left(125 \mathrm{MHz}, \mathrm{C}_{6} \mathrm{D}_{6}\right)$

192.6 (C(1)), 49.1 (C(2)), 28.9 (C(5)), 25.8 (C(8)), 22.3 (C(4, 4')), 17.2 (C(7)), $12.4(\mathrm{C}(3)),-5.0\left(\mathrm{C}\left(6,6^{\prime}\right)\right)$

IR: (neat)

2956 (s), 2929 (s), 2886 (s), 2858 (s), 2036 (s), 1471 (m), 1390 (m), 1377 (m), 1360 (m), 1303 (m), 1251 (m), 1074 (w), 1005 (w) 882 (m), 838 (s), 823 (s), 810 (s), $782(\mathrm{~s}), 742(\mathrm{~s}), 679(\mathrm{~s})$

MS: (EI, $70 \mathrm{eV})$

211.2 (28.2), 211.1 (28.2), 196.2 (58.1), 196.0 (53.9), 73.0 (100.0), 59.0 (11.4)

$\underline{\text { HRMS }}$ Calcd for $\mathrm{C}_{12} \mathrm{H}_{25} \mathrm{NSi}$ : 211.1756, found: 211.1757

\section{Preparation of 1-(1,1-Dimethylethyl)- $N$-[2-(4-methoxyphenyl)-1-propenylidene]-1,1- dimethylsilanamine $(3 \mathrm{c})$}
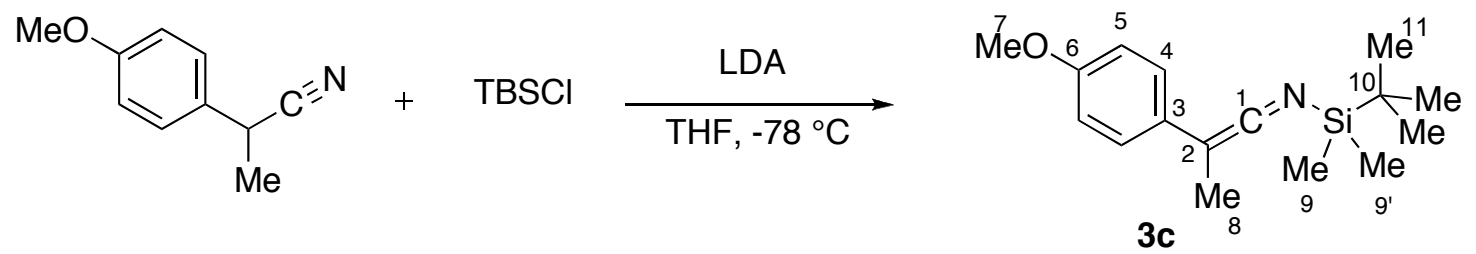

Following General Procedure 1, $i-\mathrm{Pr}_{2} \mathrm{NH}(0.703 \mathrm{~mL}, 5.0 \mathrm{mmol}, 1.0$ equiv), THF (5.5 $\mathrm{mL}), 2.6 \mathrm{M} n$-BuLi in hexanes (1.9 mL, 1.0 equiv), 2-(4-methoxyphenyl)propanenitrile (0.801 $\mathrm{g}$, $5 \mathrm{mmol})$ in $2 \mathrm{~mL}$ of THF, and a solution of TBSCl (0.904 g, 1.2 equiv, $6.0 \mathrm{mmol})$ in $2 \mathrm{~mL}$ of THF were combined to afford $1.39 \mathrm{~g}(99 \%)$ of $\mathbf{3 c}$ as yellow liquid.

Data for 3c:

${ }^{1} \mathrm{H}$ NMR: $\quad\left(500 \mathrm{MHz}, \mathrm{C}_{6} \mathrm{D}_{6}\right)$

7.11-7.07 (m, 2 H, HC(4)), 6.9-6.87 (m, 2 H, HC(5)), $3.00\left(3 \mathrm{H}, \mathrm{H}_{3} \mathrm{C}(7)\right) 1.90$ (s, 3 $\left.\mathrm{H}, \mathrm{H}_{3} \mathrm{C}(8)\right), 0.91\left(\mathrm{~s}, 9 \mathrm{H}, \mathrm{H}_{3} \mathrm{C}(11)\right), 0.11\left(\mathrm{~s}, 6 \mathrm{H}, \mathrm{H}_{3} \mathrm{C}(9)\right.$ and $\left.\mathrm{H}_{3} \mathrm{C}\left(9^{\prime}\right)\right)$

${ }^{13} \mathrm{C} \mathrm{NMR:} \quad\left(125 \mathrm{MHz}, \mathrm{C}_{6} \mathrm{D}_{6}\right)$

189.7 (C(1)), 156.3 (C(6)), 130.8 (C(3)), 124.0 (C(4)), 114.8 (C(5)), 54.8 (C(2)), $47.7(\mathrm{C}(7)), 25.5(\mathrm{C}(11)), 17.7(\mathrm{C}(10)), 12.0(\mathrm{C}(8)),-4.8\left(\mathrm{C}\left(9,9^{\prime}\right)\right)$

IR: (neat)

2953 (s), 2930 (s), 2897 (s), 2857 (s), 2034 (s), 1680 (w), 1609 (w), 1574 (w), 1509 
(s), 1471 (m), 1374 (m), 1284 (m), 1251 (m), 1179 (m), 1112 (w), 1064 (w), 1037

(s), $962(\mathrm{~m}), 840$ (s), 810 (s), 823 (s), 785 (s), $709(\mathrm{~s}), 678(\mathrm{w}), 633(\mathrm{w})$

MS: (EI, $70 \mathrm{eV})$

73 (77), 135 (20), 161 (18), 207 (16), 275 (100)

$\underline{\text { HRMS }}$ Calcd for $\mathrm{C}_{16} \mathrm{H}_{25} \mathrm{NOSi}$ : 275.1705, found: 275.1705

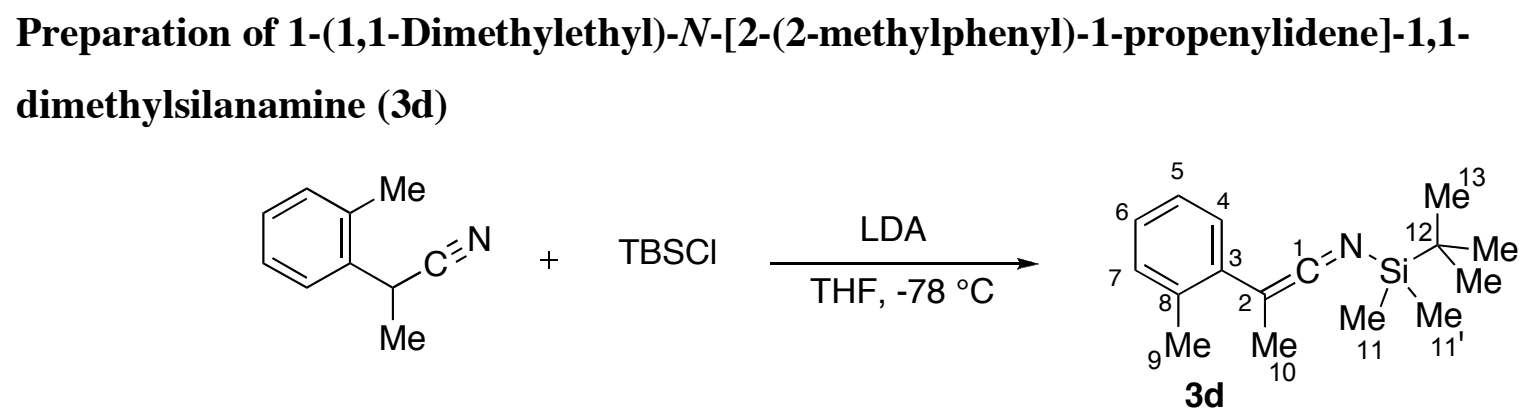

Following General Procedure 1, $i$ - $\mathrm{Pr}_{2} \mathrm{NH}(0.39 \mathrm{~mL}, 2.8 \mathrm{mmol}, 1.0$ equiv), THF ( $3 \mathrm{~mL})$, 2.41 M n-BuLi in hexanes (1.15 mL, 1.0 equiv), 2-(2-methylphenyl)propanenitrile (0.4061 g, $2.79 \mathrm{mmol})$ in $1 \mathrm{~mL}$ of THF, and a solution of TBSCl (0.904 g, 1.2 equiv, $6.0 \mathrm{mmol})$ in $1 \mathrm{~mL}$ of THF were combined to afford $646 \mathrm{mg}(89 \%)$ of $\mathbf{3 d}$ as a yellow liquid.

Data for 3d:

${ }^{1} \mathrm{H}$ NMR: $\quad\left(500 \mathrm{MHz}, \mathrm{C}_{6} \mathrm{D}_{6}\right)$

7.29-7.24 (m, $1 \mathrm{H}, \mathrm{HC}(4,5,6))$, 7.19-7.16 (m, $2 \mathrm{H}, \mathrm{HC}(4,5,6)), 7.10-7.06$ (m, $1 \mathrm{H}$ $\mathrm{HC}(7)), 2.52$ (s, $\left.3 \mathrm{H}, \mathrm{H}_{3} \mathrm{C}(9)\right), 2.08$ (s, $\left.3 \mathrm{H}, \mathrm{H}_{3} \mathrm{C}(10)\right), 0.95$ (s, $9 \mathrm{H}, \mathrm{H}_{3} \mathrm{C}(13)$ ), 0.13 (s, $6 \mathrm{H}, \mathrm{H}_{3} \mathrm{C}(11)$ and $\mathrm{H}_{3} \mathrm{C}\left(11^{\prime}\right)$ )

${ }^{13} \mathrm{C}$ NMR: $\quad\left(125 \mathrm{MHz}, \mathrm{C}_{6} \mathrm{D}_{6}\right)$

$185.2(\mathrm{C}(1)), 137.0(\mathrm{C}(8)), 134.1(\mathrm{C}(3)), 130.9(\mathrm{C}(4,5,6,7)), 126.6(\mathrm{C}(4,5,6,7))$, $124.8(\mathrm{C}(4,5,6,7)), 124.0\left(\mathrm{HC}(4,5,6,7), 45.9(\mathrm{C}(2)), 25.5\left(\mathrm{H}_{3} \mathrm{C}(13), 22.2(\mathrm{C}(9))\right.\right.$, $17.7(\mathrm{C}(12)), 15.4(\mathrm{C}(10)),-5.3\left(\mathrm{C}\left(11,11^{\prime}\right)\right)$

MS: (EI, $70 \mathrm{eV})$

73 (100), 119 (30), 145 (16), 202 (37), 259 (85)

HRMS Calcd for $\mathrm{C}_{16} \mathrm{H}_{25} \mathrm{NSi}$ : 259.1756, found: 259.1758 


\section{General Procedure 2. Addition of Silyl Ketene Imines to Benzaldehyde.}

\section{Preparation of (2R,3S)-3-Hydroxy-2-methyl-2,3-diphenylpropanenitrile (6aa) (Table 1, entry 1)}

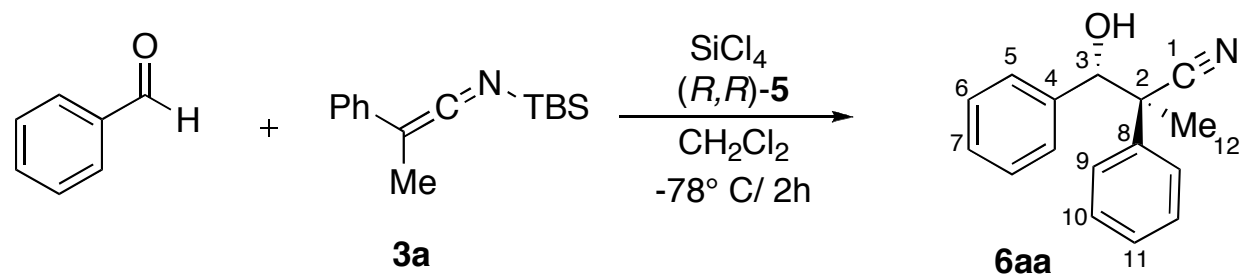

To a flame-dried, 10-mL, Schlenk flask equipped with a magnetic stir bar, a thermocouple, a gas inlet tube and a septum were added $(R, R)-5$ (42 mg, $0.05 \mathrm{mmol}, 0.05$ equiv), benzaldehyde (156 mg, $1.0 \mathrm{mmol})$ and $\mathrm{CH}_{2} \mathrm{Cl}_{2}(4.0 \mathrm{~mL}, 0.25 \mathrm{M}$ in aldehyde). The solution was cooled to $-78{ }^{\circ} \mathrm{C}$ (internal temp) in a dry ice-acetone bath and $126 \mu \mathrm{L}$ of $\mathrm{SiCl}_{4}(1.1 \mathrm{mmol}, 1.1$ equiv) was added to the reaction mixture with stirring. The resulting bright-yellow solution was allowed to stir for $5 \mathrm{~min}$ at $-78^{\circ} \mathrm{C}$ and then $2 \mathrm{~mL}$ of a $0.6 \mathrm{M}$ solution of silyl ketene imine 3a in $\mathrm{CH}_{2} \mathrm{Cl}_{2}$ (1.2 mmol, 1.2 equiv) was added dropwise via syringe over $5 \mathrm{~min}$. The resulting reaction mixture was allowed to stir at $-78{ }^{\circ} \mathrm{C}$ for $2 \mathrm{~h}$ and was then quenched by pouring the cold solution into a $125-\mathrm{mL}$ Erlenmeyer flask containing a rapidly stirring solution of sat. aq. KF (20 $\mathrm{mL})$ and sat. aq. $\mathrm{NaHCO}_{3}(20 \mathrm{~mL})$ solutions. The biphasic mixture was vigorously stirred for 3 $\mathrm{h}$ at ambient temperature and then was filtered through a pad of Celite (ca. $7 \mathrm{~g}$ ) using a $60 \mathrm{~mL}$, coarse glass frit, Büchner funnel. The filtrate was transferred to a $125-\mathrm{mL}$ separatory funnel and the organic layer was isolated. The aqueous layer was extracted with $\mathrm{CH}_{2} \mathrm{Cl}_{2}(3 \times 25 \mathrm{~mL})$ and then the organic layers were combined and dried over $\mathrm{MgSO}_{4}$ (ca. $3 \mathrm{~g}$ ). The solution was filtered and concentrated in vacuo $\left(23^{\circ} \mathrm{C}, 14 \mathrm{mmHg}\right)$ and the resulting residue was purified by column chromatography (silica gel (30 g), $3 \mathrm{~cm}$ diam, hexanes/EtOAc, 4:1) to afford $212 \mathrm{mg}$ of a white solid, which was recrystallized from hot EtOAc (ca. $0.5 \mathrm{~mL}$ ) and hexanes (ca. $1-0.5 \mathrm{~mL})$ to yield $206 \mathrm{mg}(87 \%)$ of $6 \mathbf{a a}$ as white needles.

\section{Data for 6aa:}

mp: $93-94^{\circ} \mathrm{C}$

${ }^{1} \mathrm{H}$ NMR: $\quad\left(500 \mathrm{MHz}, \mathrm{CDCl}_{3}\right)$

$7.49(\mathrm{dt}, J=8.3,1.7,2 \mathrm{H}, \mathrm{HC}(10))$, 7.43-7.37 (m, $3 \mathrm{H}, \mathrm{HC}(10,11))$, 7.37-7.30 (m, 5 H, $\mathrm{HC}(5,6,7)), 4.87$ (s, $1 \mathrm{H}, \mathrm{HC}(3)), 2.24$ (br. s, $1 \mathrm{H}, \mathrm{OH}), 1.61$ (s, $3 \mathrm{H}, \mathrm{H}_{3} \mathrm{C}(12)$ ) 
${ }^{13} \mathrm{C} \mathrm{NMR:} \quad\left(125 \mathrm{MHz}, \mathrm{CDCl}_{3}\right)$

137.8 (C(4)), $137.4(\mathrm{C}(8)), 128.7$ (C(7, 10)), 128.3 (C(11)), $128.0(\mathrm{C}(5)), 127.5$

(C(6)), 126.7 (C(9)), 121.8 (C(1)), 79.6 (C(3)), 49.4 (C(2)), 22.5 (C(12))

IR: $\left(\mathrm{CHCl}_{3}\right)$

3454 (m), 3090 (w), 2991 (w), 2882 (w), 1601 (w), 1496 (m), 1454 (m), 1447 (m),

1380 (m), 1199 (m), 1159 (m), 1088 (m), 1076 (m), 1065 (m), 1045 (s), 1027 (s),

$911(\mathrm{~m}), 778(\mathrm{~m}), 756(\mathrm{~m}), 717(\mathrm{~s}), 699(\mathrm{~s}), 671(\mathrm{~m}), 657(\mathrm{~m}), 610(\mathrm{~m})$

MS: $\mathrm{CI}\left(\mathrm{CH}_{4}\right)$

238 (M+H, 6), 221 (11), 220 (56), 160 (13), 133 (13), 132 (100), 131 (26), 107

(53), 105 (66)

Opt. Rot.: $[\alpha]_{\mathrm{D}}^{24} 19.8(\mathrm{c}=1.08, \mathrm{EtOH})$

TLC: $\quad R_{f} 0.17$ (hexanes/EtOAc, 4:1) [4-anisaldehyde]

SFC: $(2 R, 3 S)-6 \mathbf{a a}, t_{\mathrm{R}} 3.83 \mathrm{~min}(98.5 \%)$; (2S,3R)-6aa, $t_{\mathrm{R}} 4.89 \mathrm{~min}(1.47 \%)$, (Chiralpak AS, 125 bar, $13 \% \mathrm{MeOH}$ in $\mathrm{CO}_{2}, 3 \mathrm{~mL} / \mathrm{min}$ )

Analysis: $\mathrm{C}_{16} \mathrm{H}_{15} \mathrm{NO}(237.3)$

Calcd: C, 80.98; H, 6.37; N, 5.90\%

Found: $\quad$ C, 80.70; H, 6.35; N, 5.97\%

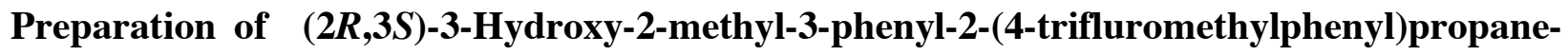 nitrile (6ab) (Table 1, entry 2)}
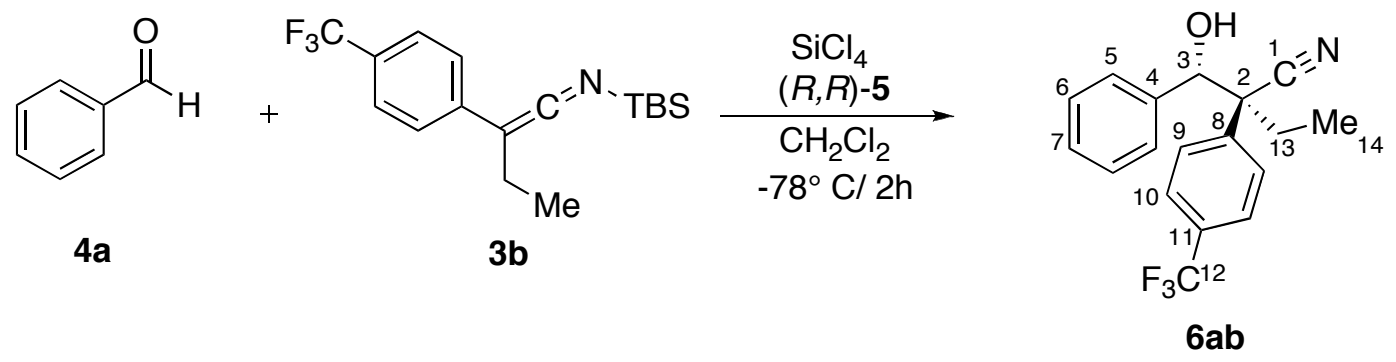

Following General Procedure 2, $106 \mathrm{mg}$ of benzaldehyde (1.0 mmol, 1.0 equiv) was combined with $42 \mathrm{mg}$ of $(R, R)-5\left(0.05 \mathrm{mmol}, 0.05\right.$ equiv), $126 \mu \mathrm{L}$ of $\operatorname{SiCl}_{4}(1.1 \mathrm{mmol}, 1.1$ equiv), and $0.86 \mathrm{~mL}$ of a $1.4 \mathrm{M}$ solution of silyl ketene imine $3 \mathbf{b}$ (1.2 mmol, 1.2 equiv) to yield after column chromatography $\left(\mathrm{SiO}_{2}(32 \mathrm{~g}) 3 \mathrm{~cm}\right.$ diam., hexanes/EtOAc 5:1) $258 \mathrm{mg}(80 \%)$ of 6ab as a white solid, and $11 \mathrm{mg}(3 \%)$ of the diastereomer as an oil. The white solid was 
crystallized from hot $i-\mathrm{Pr}_{2} \mathrm{O}(2 \mathrm{~mL})$ and hexanes $(2 \mathrm{~mL})$ to yield $234 \mathrm{mg}(73 \%)$ of $\mathbf{6 a b}$ as white needles.

Data for 6ab:

mp: $\quad 111-116^{\circ} \mathrm{C}$

'H NMR: $\quad\left(500 \mathrm{MHz}, \mathrm{CDCl}_{3}\right)$

7.67-7.62 (m, $2 \mathrm{H}, \mathrm{HC}(10))$, 7.58-7.54 (m, $2 \mathrm{H}, \mathrm{HC}(9))$, 7.38-7.31 (m, $3 \mathrm{H}, \mathrm{HC}(7,6))$, 7.30-7.27 (m, $2 \mathrm{H}, \mathrm{HC}(5)), 4.93$ (s, $1 \mathrm{H}, \mathrm{HC}(3)), 2.37$ (br. s, $1 \mathrm{H}, \mathrm{OH})$ ), 2.11 (dq, $J=$ 7.4, 14.8, $\left.1 \mathrm{H}, \mathrm{H}_{2} \mathrm{C}(13)\right), 1.89$ (dq, $\left.J=7.4,14.8,1 \mathrm{H}, \mathrm{H}_{2} \mathrm{C}\left(13^{`}\right)\right), 0.83$ (t, $J=7.4,3$ $\left.\mathrm{H}, \mathrm{H}_{3} \mathrm{C}(14)\right)$

${ }^{13} \mathrm{C}$ NMR: $\quad\left(125 \mathrm{MHz}, \mathrm{CDCl}_{3}\right)$ 139.5 (C(4,8)), $138.1(\mathrm{C}(4,8)), 130.4$ (q, J = 33.0, C(11)), 129.1 (C(7)), $120.2(\mathrm{C}(6))$, 127.9 (C(9)), 125.5 (q, $J=3.5, \mathrm{C}(10)), 123.8$ (q, $J=272.4, \mathrm{C}(12)), 120.0(\mathrm{C}(1))$, $79.3(\mathrm{C}(3)), 56.2(\mathrm{C}(2)), 28.8(\mathrm{C}(13)), 9.2(\mathrm{C}(14))$

IR: $(\mathrm{KBr}$ pellet $)$ $3563(\mathrm{~m}), 3088$ (w), 3067 (w), 3052 (w), 3037 (w), $2986(\mathrm{w}), 2949$ (w), $2891(\mathrm{w})$, 2241 (w), 1952 (w), 1915 (w), 1620 (w), 1453 (m), 1413 (m), 1325 (s), 1164 (s), 1137 (s), 1116 (s), 1067 (s), 1019 (s), 847 (m), 712 (m), 698 (m), 686 (m), $624(\mathrm{~m})$ MS: (ESI)

102 (27), 214 (12), 275 (14), 302 (22), $342\left(\mathrm{M}^{+}+\mathrm{Na}, 37\right), 512$ (100), 516 (23), 658 (31), 666 (39), 667 (15), 826 (11)

HRMS Calcd for $\mathrm{C}_{18} \mathrm{H}_{16} \mathrm{NOF}_{3} \mathrm{Na}$ : 342.1082 , found: 342.1096

Opt. Rot.: $[\alpha]_{\mathrm{D}}{ }^{24} 58.5\left(\mathrm{c}=0.515, \mathrm{CHCl}_{3}\right)$

TLC: $\quad R_{f} 0.32$ (hexanes/EtOAc, 4:1) [UV (254 nm)]

SFC: $\quad(2 R, 3 S)-\mathbf{6 a b}, t_{\mathrm{R}} 7.0 \mathrm{~min}(99.5 \%) ;(2 S, 3 R)-\mathbf{6 a b}, t_{\mathrm{R}} 6.6 \min (0.5 \%)$ (Chiralpak OD, 125 bar, $5 \% \mathrm{MeOH}$ in $\left.\mathrm{CO}_{2}, 2.5 \mathrm{~mL} / \mathrm{min}\right)$

Analysis: $\mathrm{C}_{18} \mathrm{H}_{16} \mathrm{~F}_{3} \mathrm{NO}(319.32)$

Calcd: C, 67.70; H, 5.05; N, 4.39\%

Found: $\quad$ C, $67.98 ; \quad H, 4.89 ; \quad \mathrm{N}, 4.52 \%$ 


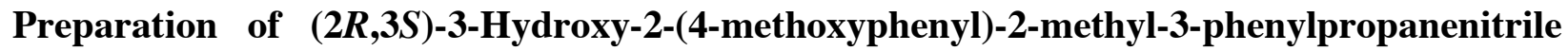
(6ac) (Table 1, entry 3)
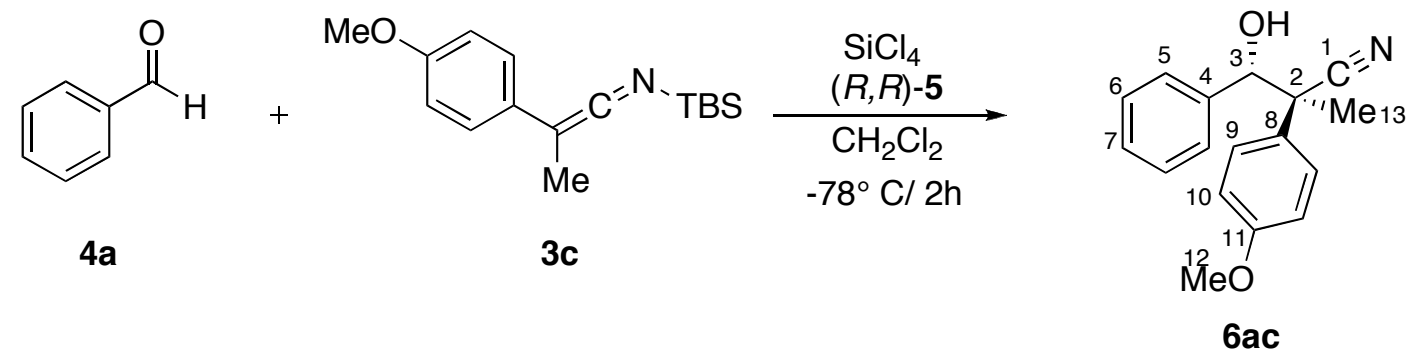

Following General Procedure 2, $106 \mathrm{mg}$ of benzaldehyde (1.0 mmol, 1.0 equiv) was combined with $42 \mathrm{mg}$ of $(R, R)-5$ (0.05 mmol, 0.05 equiv), $126 \mu \mathrm{L}$ of $\mathrm{SiCl}_{4}(1.1 \mathrm{mmol}, 1.1$ equiv), and $0.86 \mathrm{~mL}$ of a $1.4 \mathrm{M}$ solution of silyl ketene imine 3c (1.2 mmol, 1.2 equiv) to yield after column chromatography $\left(\mathrm{SiO}_{2}(30 \mathrm{~g}), 3 \mathrm{~cm}\right.$ diam., gradient from $\mathrm{CH}_{2} \mathrm{Cl}_{2} /$ hexanes 7:1 to neat $\mathrm{CH}_{2} \mathrm{Cl}_{2}$ to $\mathrm{CH}_{2} \mathrm{Cl}_{2} /$ EtOAc 7:1) $295 \mathrm{mg}$ of $\mathbf{6 a c}$ as a white solid, which was crystallized from hot isopropyl acetate $(1 \mathrm{~mL})$ and an equal volume of hexanes $(1 \mathrm{~mL})$ to yield $240 \mathrm{mg}(90 \%)$ of 6ac as white needles.

Data for 6ac:

mp: $\quad 153-154^{\circ} \mathrm{C}$

${ }^{1} \mathrm{H}$ NMR: $\quad\left(500 \mathrm{MHz}, \mathrm{CDCl}_{3}\right)$

7.40-7.36 (m, $2 \mathrm{H}, \mathrm{HC}(9))$, 7.35-7.32 (m, $3 \mathrm{H}, \mathrm{HC}(7,6))$, 7.31-7.28 (m, $2 \mathrm{H}$, $\mathrm{HC}(5))$, 6.94-6.90 (m, 2 H, HC(10)), 4.82 (s, 1 H, HC(3)), 3.83 (s, 3 H, H $\mathrm{H}_{3} \mathrm{C}(12)$ ), $1.58\left(\mathrm{~s}, 3 \mathrm{H}, \mathrm{H}_{3} \mathrm{C}(13)\right)$

${ }^{13} \mathrm{C} \mathrm{NMR:} \quad\left(125 \mathrm{MHz}, \mathrm{CDCl}_{3}\right)$

159.5 (C(11)), 137.8 (C(4)), 129.3 (C(8)), 128.8 (C(7)), 128.1 (C(6)), 128.0 (C(9)), 127.6 (C(5)), 122.0 (C(1)), 114.1 (C(10)), 79.9 (C(10)), 55.3 (C(12)), $48.7(\mathrm{C}(2))$

IR: $(\mathrm{KBr}$ pellet)

3482 (s), 3086 (w), 3068 (w), 3038 (w), 2992 (m), 2946 (m), 2847 (w), 2242 (w), 1612 (w), 1585 (w), 1514 (s), 1453 (m), 1408 (w), 1379 (m), 1300 (m), 1248 (s), $1189(\mathrm{~m}), 1040$ (m), 1026 (m), 885 (w), 828 (m), 800 (m), $710(\mathrm{~s}), 643$ (w), 609 (w)

MS: (ESI) $290\left(\mathrm{M}^{+}+\mathrm{Na}, 100\right), 291(17)$ 
$\underline{\text { HRMS }}$ Calcd for $\mathrm{C}_{17} \mathrm{H}_{17} \mathrm{NO}_{2} \mathrm{Na}: 290.1157$, found: 290.1154

Opt. Rot.: $[\alpha]_{\mathrm{D}}^{24} 19.4\left(\mathrm{c}=0.495, \mathrm{CHCl}_{3}\right)$

TLC: $\quad R_{f} 0.15$ (hexanes/EtOAc, 4:1) [UV (254 nm)]

SFC: $\quad(2 R, 3 S)$-6ac, $t_{\mathrm{R}} 9.4 \min (97.8 \%) ;(R, R) /(S, S)-6 \mathbf{6 a c}, t_{\mathrm{R}} 7.8 \mathrm{~min}(0.8 \%) ;(R, R) /(S, S)$ 6ac, $t_{\mathrm{R}} 8.3 \min (0.5 \%) ;(2 S, 3 R)-6 \mathbf{a c}, t_{\mathrm{R}} 10.5 \min (0.9 \%)$ (Chiralpak AD, 125 bar, $40{ }^{\circ} \mathrm{C}, 8 \% \mathrm{MeOH}$ in $\mathrm{CO}_{2}, 2.5 \mathrm{~mL} / \mathrm{min}$ )

Analysis: $\quad \mathrm{C}_{17} \mathrm{H}_{17} \mathrm{NO}_{2}(267.32)$

Calcd: $\quad$ C, 76.38; H, 6.41; N, 5.24\%

Found: $\quad$ C, $76.58 ; \quad H, 6.45 ; \quad$ N, $5.36 \%$

Preparation of $(2 R, 3 S)$-3-Hydroxy-2-methyl-2-(2-methylphenyl)-3-phenylpropanenitrile (6ad) (Table 1, entry 4)
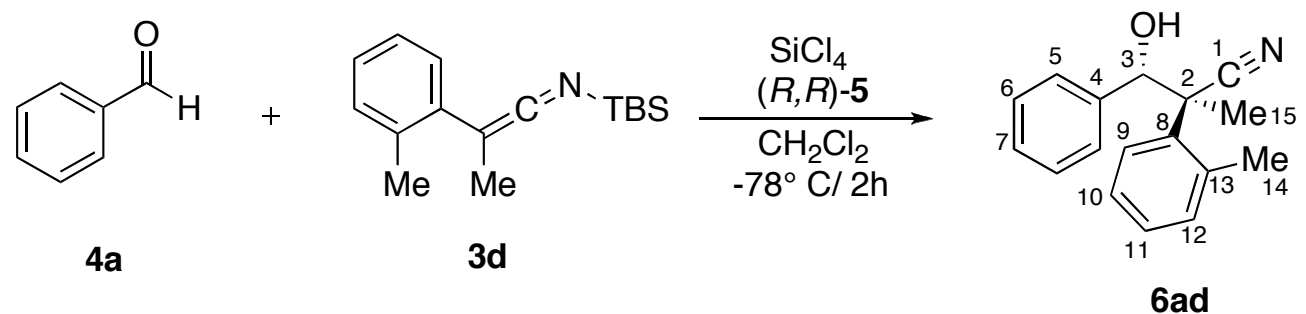

Following General Procedure 2, $106 \mathrm{mg}$ of benzaldehyde (1.0 mmol, 1.0 equiv) was combined with $42 \mathrm{mg}$ of $(R, R)-5$ (0.05 mmol, 0.05 equiv), $126 \mu \mathrm{L}$ of $\mathrm{SiCl}_{4}(1.1 \mathrm{mmol}, 1.1$ equiv), and $0.86 \mathrm{~mL}$ of a $1.4 \mathrm{M}$ solution of silyl ketene imine $\mathbf{3 d}$ (1.2 mmol, 1.2 equiv) to yield after column chromatography ( $\mathrm{SiO}_{2}(32 \mathrm{~g}) 3 \mathrm{~cm}$ diam., hexanes/EtOAc 4:1 ) $222 \mathrm{mg}$ (88\%) of 6ad as a white solid. A sample (3.6 mg) was taken for derivatization and determination of er (see compound 7ad). The white solid was crystallized from $i$ - $\operatorname{Pr}_{2} \mathrm{O}(2 \mathrm{~mL})$ and 20 drops of hexanes to yield $187 \mathrm{mg}(74 \%)$ of $\mathbf{6 a d}$ as white needles.

Data for 6ad:

mp: $\quad 121-122{ }^{\circ} \mathrm{C}$

'H NMR: $\quad\left(500 \mathrm{MHz}, \mathrm{CDCl}_{3}\right)$

7.27-7.24 (m, $3 \mathrm{H}, \mathrm{HC}(6,12))$, 7.23-7.19 (m, $2 \mathrm{H}, \mathrm{HC}(11,7))$, 7.14-7.09 (m, $1 \mathrm{H}$, $\mathrm{HC}(10))$, 7.03-7.00 (m, $3 \mathrm{H}, \mathrm{HC}(5,9)), 5.27$ (s, $1 \mathrm{H}, \mathrm{HC}(3)$ ), 2.70 (s, $3 \mathrm{H}, \mathrm{H}_{3} \mathrm{C}(14)$ ), $1.60\left(\mathrm{~s}, 3 \mathrm{H}, \mathrm{H}_{3} \mathrm{C}(15)\right)$ 
${ }^{13} \mathrm{C} \mathrm{NMR:} \quad\left(125 \mathrm{MHz}, \mathrm{CDCl}_{3}\right)$

$137.3(\mathrm{C}(8)), 135.6(\mathrm{C}(4,13)), 134.2$ (C(4,13)), $132.8(\mathrm{C}(12)), 128.4(\mathrm{C}(11,7))$, 128.2 (C(11,7)), 127.7 (C(9)), 127.49 (C(6,5)), 127.46 (C(6,5)), 126.4 (C(10)), $122.7(\mathrm{C}(1)), 76.0(\mathrm{C}(3)), 47.3(\mathrm{C}(2)), 21.9(\mathrm{C}(14)), 20.8(\mathrm{C}(15))$

IR: $(\mathrm{KBr}$ pellet $)$

3484 (s), 3059 (w), 3034 (w), 3005 (w), 2986 (w), 2939 (w), 2237 (m), 1956 (w), 1488 (m), 1453 (m), 1406 (w), 1389 (w), 1375 (m), 1329 (w), 1292 (w), 1245 (w), 1197 (m), 1088 (w), 1059 (m), 1033 (m), 915 (m), 771 (m), 748 (m), 721 (s), 711 (s)

MS: (ESI)

146 (100), 147 (12), 207 (18), 234 (97), 235 (27), 258 (13), 169 (26), 274 (M+Na, 34), 345 (12)

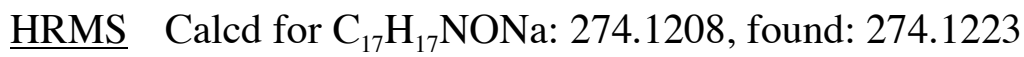

Opt. Rot.: $[\alpha]_{\mathrm{D}}{ }^{24}-59.5\left(\mathrm{c}=0.495, \mathrm{CHCl}_{3}\right)$

TLC: $\quad R_{f} 0.28$ (hexanes/EtOAc, 4:1) [UV (254 nm)]

Analysis: $\mathrm{C}_{17} \mathrm{H}_{17} \mathrm{NO}(251.32)$

Calcd: C, 81.24; H, 6.82; N, 5.57\%

Found: $\quad$ C, 81.00; H, 6.79; N, $5.70 \%$ 


\section{Derivatization of Nitrile 6ad with 3,5-Dinitrobenzoyl Chloride.}

Preparation of (1S,2R)-2-Cyano-1-phenyl-2-(2-methylphenyl)propyl-3,5-Dinitrobenzoate (7ad)
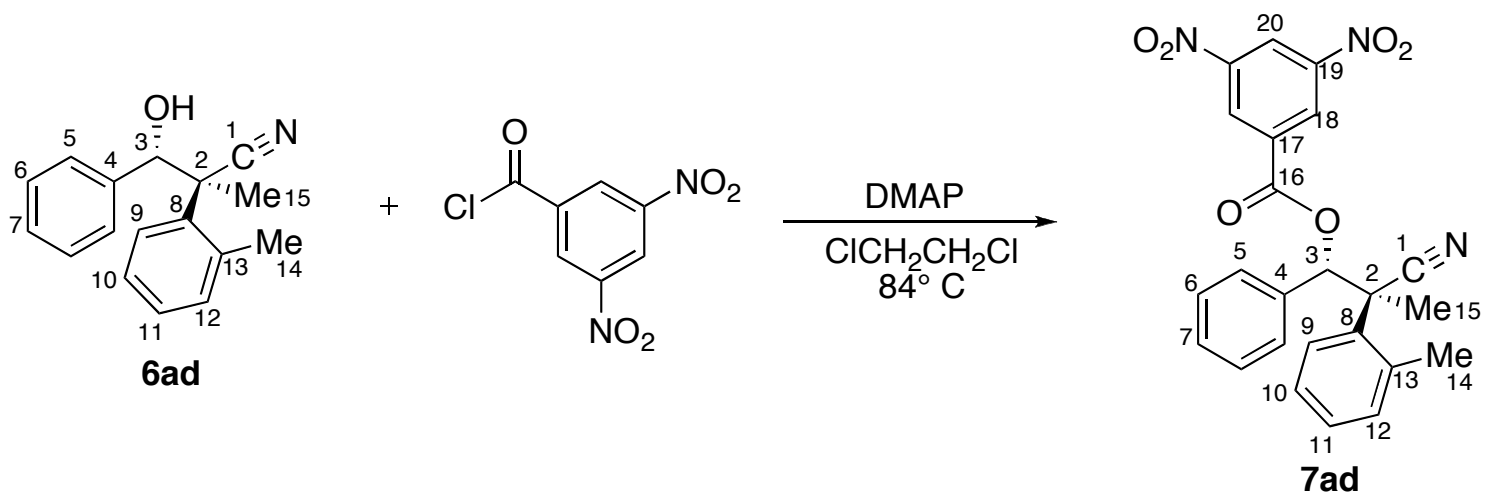

A 10-mL, round-bottomed flask equipped with a condenser, inert gas inlet, and magnetic stir bar was flame dried. The flask was charged with 6ad (0.25 mmol, $62.8 \mathrm{mg}, 1.0$ equiv), dry 1,2-dichloroethane (2.5 mL), 4-dimethylaminopyridine $(61.1 \mathrm{mg}, 0.5 \mathrm{mmol}, 2.0$ equiv), and 3,5dinitrobenzoylchloride ( $86.4 \mathrm{mg}, 0.375 \mathrm{mmol}, 1.5$ equiv), under Ar. The mixture was heated to reflux for $6.5 \mathrm{hr}$, then was stirred overnight at room temperature. The resulting mixture was poured into a $125-\mathrm{mL}$ separatory funnel and the flask was rinsed with $5 \mathrm{~mL}$ of $\mathrm{CH}_{2} \mathrm{Cl}_{2}$ to dissolve the solids. Water $(15 \mathrm{~mL})$, and $\mathrm{Et}_{2} \mathrm{O}(15 \mathrm{~mL})$ were added to the separatory funnel and the mixture washed $3 \times 15 \mathrm{~mL} \mathrm{Et}_{2} \mathrm{O}$. The combined organic layers were washed with aq. $\mathrm{CuSO}_{4}$ solution $(10 \mathrm{~mL})$ and brine $(2 \times 10 \mathrm{~mL})$. The organic layer was dried over $\mathrm{MgSO}_{4}$, then was filtered and concentrated in vacuo $\left(23^{\circ} \mathrm{C}, 15 \mathrm{mmHg}\right)$. The residue was purified by column chromatography $\left(\mathrm{SiO}_{2}(15 \mathrm{~g}), 2 \mathrm{~cm}\right.$ diameter column, gradient from $\mathrm{CH}_{2} \mathrm{Cl}_{2} /$ hexanes 3:2, to $\mathrm{CH}_{2} \mathrm{Cl}_{2} /$ hexanes 3:1) to yield $7 \mathbf{a d} 78 \mathrm{mg}$, (70\%) as a white solid, which was recrystallized from EtOAc $(0.5 \mathrm{~mL})$ and hexanes $(0.5 \mathrm{~mL})$ to afford $7 \mathbf{a d}$ as white needles $(70 \mathrm{mg}, 63 \%)$.

Data for 7ad:

mp: $190-195^{\circ} \mathrm{C}$

${ }^{1} \mathrm{H}$ NMR: $\quad\left(500 \mathrm{MHz}, \mathrm{CDCl}_{3}\right)$

$9.28(\mathrm{t}, J=2.1,1 \mathrm{H}, \mathrm{HC}(20)), 9.18(\mathrm{~d}, J=2.1,2 \mathrm{H}, \mathrm{HC}(18)), 7.35(\mathrm{~m}, 1 \mathrm{H}$, $\mathrm{HC}(7,6,5,9,10,11)), 7.33-7.29(\mathrm{~m}, 4 \mathrm{H}, \mathrm{HC}(7,6,5,9,10)), 7.18-7.13(\mathrm{~m}, 4 \mathrm{H}$, $\mathrm{HC}(7,6,5,9,10)), 6.70$ (s, $1 \mathrm{H}, \mathrm{HC}(3)), 2.81$ (s, $\left.3 \mathrm{H}, \mathrm{H}_{3} \mathrm{C}(14)\right), 1.90$ (s, $\left.3 \mathrm{H}, \mathrm{H}_{3} \mathrm{C}(15)\right)$ 
${ }^{13} \mathrm{C} \mathrm{NMR:} \quad\left(125 \mathrm{MHz}, \mathrm{CDCl}_{3}\right)$

161.1 (C(16)), 148.8 (C(19)), 136.4 (C(4,8,13,17)), 133.2 (C(12)), 133.02 (C(4,8,13,17)), 132.97 (C(4,8,13,17)), 132.8 (C(4,8,13,17)), 129.46 (C(18)), 129.1 $(\mathrm{C}(7,6,5,9,10,11)), 128.3(\mathrm{C}(7,6,5,9,10,11)), 127.54 \quad(\mathrm{C}(7,6,5,9,10,11)), 127.52$ $(\mathrm{C}(7,6,5,9,10,11)), 126.6(\mathrm{C}(7,6,5,9,10,11)), 122.9$ (C(20)), $121.2(\mathrm{C}(1)), 78.3$ (C(3)), $46.0(\mathrm{C}(2)), 21.8(\mathrm{C}(14,15)), 21.7(\mathrm{C}(14,15))$

IR: $(\mathrm{KBr}$ pellet)

3113 (m), 3091 (w), 2997 (w), 2234 (w), 1742 (s), 1629 (m), 1544 (s), 1456 (m), 1347 (s), 1329 (m), 1269 (s), 1207 (w), 1156 (s), 1076 (w), 1032 (w), 981 (s), 920 (m), 912 (m), 771 (m), 764 (m), 757 (m), 732 (s), 719 (s), 703 (s), 632 (m)

MS: (ESI)

234 (12), $468\left(\mathrm{M}^{+}+\mathrm{Na}, 100\right), 469$ (18)

Opt. Rot.: $[\alpha]_{\mathrm{D}}{ }^{24}-99.9\left(\mathrm{c}=0.485, \mathrm{CHCl}_{3}\right)$

TLC: $\quad R_{f} 0.33$ (hexanes/EtOAc, 4:1) [UV (254 nm)]

HPLC: $\quad(2 R, 3 S)-7 \mathbf{a d}, t_{\mathrm{R}} 38.2 \min (85.2 \%)$; $(R, R) /(S, S)-7 \mathbf{a d}, t_{\mathrm{R}} 26.7 \min (10.1 \%)$; $(2 R, 3 S)-$ 7ad, $t_{\mathrm{R}} 33.5 \min (5.1 \%)$ (Rexchrom Pirkle Covalent S-N1N-Naphthylleucine, $1 \mathrm{~mL}$ /min from 0 to $20 \mathrm{~min}, 0.9 \mathrm{~mL} / \mathrm{min}$ at $30 \mathrm{~min}, 20 \% i-\mathrm{PrOH}$ in hexane from 0 to 20 min, then a gradient to $50 \%$ over $10 \mathrm{~min}$.)

Analysis: $\mathrm{C}_{24} \mathrm{H}_{19} \mathrm{~N}_{3} \mathrm{O}_{6}$ (445.2)

Calcd: $\quad$ C, 64.72; H, 4.30; N, $9.43 \%$

Found: $\quad$ C, 64.99; H, 4.20; N, 9.39\% 


\section{General Procedure 3. Addition of Different Silyl Ketene Imines to 2-Naphthaldehyde}

Preparation of (2R,3S)-3-Hydroxy-2-methyl-3-(2-naphthyl)-2-phenylpropanenitrile (6ba) (Table 2, entry 1)

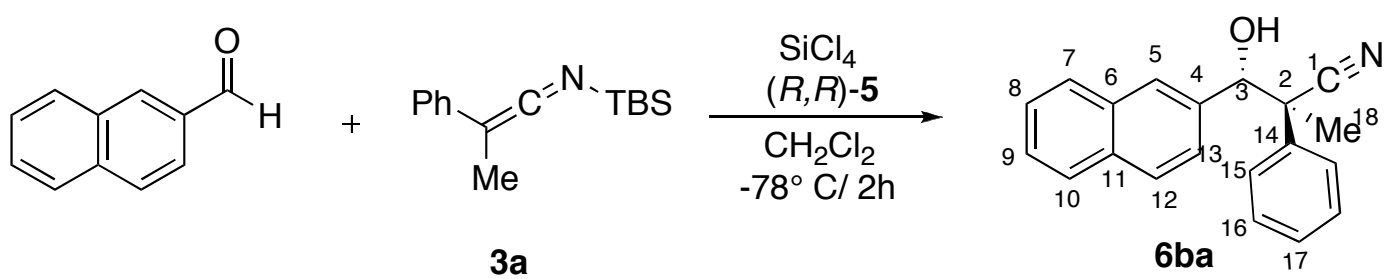

To a flame-dried, 10-mL, Schlenk flask equipped with a magnetic stir bar, a thermocouple, a gas inlet tube and a septum were added $(R, R)-5$ (42 mg, $0.05 \mathrm{mmol}, 0.05$ equiv), 2-naphthaldehyde (156 mg, $1.0 \mathrm{mmol})$ and $\mathrm{CH}_{2} \mathrm{Cl}_{2}(4.0 \mathrm{~mL}, 0.25 \mathrm{M}$ in aldehyde). The solution was stirred, cooled to $-78{ }^{\circ} \mathrm{C}$ (internal temp) in a dry ice-acetone bath and $126 \mu \mathrm{L}$ of $\mathrm{SiCl}_{4}(1.1$ mmol, 1.1 equiv) was added to the reaction mixture. The resulting bright yellow solution was allowed to stir for $5 \mathrm{~min}$ at $-78^{\circ} \mathrm{C}$ and then $0.85 \mathrm{~mL}$ of a $1.41 \mathrm{M}$ solution of silyl ketene imine 3a in $\mathrm{CH}_{2} \mathrm{Cl}_{2}(1.2 \mathrm{mmol}, 1.2$ equiv) was added dropwise via syringe over $5 \mathrm{~min}$. The resulting reaction mixture was allowed to stir at $-78{ }^{\circ} \mathrm{C}$ for $2 \mathrm{~h}$ and was then quenched by pouring the cold solution into a $125-\mathrm{mL}$ Erlenmeyer flask containing a rapidly stirring solution of sat. aq. KF (20 $\mathrm{mL})$ and sat. aq. $\mathrm{NaHCO}_{3}$ solutions $(20 \mathrm{~mL})$. The biphasic mixture was vigorously stirred for 3 $\mathrm{h}$ at ambient temperature and then was filtered through a pad of Celite (ca. $7 \mathrm{~g}$ ) using a $60 \mathrm{~mL}$, coarse glass fritted, Büchner funnel. The filtrate was transferred to a $250-\mathrm{mL}$ separatory funnel and the organic layer was separated. The aqueous layer was extracted with $\mathrm{CH}_{2} \mathrm{Cl}_{2}(3 \times 30 \mathrm{~mL})$ and then the organic layers were combined and washed with brine $(1 \times 40 \mathrm{~mL})$. The aqueous washes were back-extracted with EtOAc $(1 \mathrm{x} 40 \mathrm{~mL})$ and then the organic layers were combined and dried over $\mathrm{MgSO}_{4}$ (ca. $2 \mathrm{~g}$ ), filtered and concentrated in vacuo $\left(25^{\circ} \mathrm{C}, 15 \mathrm{mmHg}\right.$ ). The residue was purified by column chromatography $\left(\mathrm{SiO}_{2}(30 \mathrm{~g}), 3 \mathrm{~cm}\right.$ diam., $\mathrm{CH}_{2} \mathrm{Cl}_{2} /$ hexanes, 9:1 to $\left.\mathrm{CH}_{2} \mathrm{Cl}_{2} / \mathrm{EtOAc}, 9: 1\right)$ to afford $252 \mathrm{mg}(88 \%)$ of $\mathbf{6 b a}$ as a white solid.

Data for 6ba:

mp: $\quad 133-135^{\circ} \mathrm{C}$

${ }^{1} \mathrm{H}-\mathrm{NMR}: \quad\left(500 \mathrm{MHz}, \mathrm{CDCl}_{3}\right)$

7.85-7.51 (m, 4 H, HC(aryl)), 7.51-7.48 (m, 4 H, 3 X HC(aryl) and HC(15)), 7.43-7.38 (m, 4 H, 1 x HC(aryl), HC(16), and HC(17)), 5.03 (s, $1 \mathrm{H}, \mathrm{HC}(3)), 2.47$ (br s, $1 \mathrm{H}, \mathrm{OH}), 1.64$ (s, $3 \mathrm{H}, \mathrm{H}_{3} \mathrm{C}(18)$ ) 
${ }^{13} \mathrm{C}-\mathrm{NMR}: \quad\left(126 \mathrm{MHz}, \mathrm{CDCl}_{3}\right)$

$137.6(\mathrm{C}(15)), 135.4(\mathrm{C}(4)), 133.6(\mathrm{C}(6,11)), 132.8$ (C(6, 11)), $129.0(\mathrm{C}(16,17))$, 128.6 (C(aryl)), 128.4 (C(aryl)), 127.9 (C(aryl)), 127.8 (C(aryl)), 127.2 (C(5, 13)), $126.9(\mathrm{C}(16,17)), 126.6$ (C(aryl)), 126.5 (C(aryl)), 125.1 (C(5,13)), $122.0(\mathrm{C}(1))$, $80.1(\mathrm{C}(3)), 49.6(\mathrm{C}(2)), 22.6(\mathrm{C}(18))$

IR: $\quad(\mathrm{KBr}$ pellet $)$ 3434 (s), 3058 (m), 3023 (m), 2987 (m), 2883 (m), 2246 (m), 1599 (m), 1506 (m), 1497 (s), 1446 (s), 1381 (m), 1272 (m), 1168 (m), 1074 (m), 1048 (s), 1027 (s), $898(\mathrm{~m}), 863(\mathrm{~m}), 827$ (s), $776(\mathrm{~s}), 754(\mathrm{~s}), 723$ (s), $696(\mathrm{~s})$

MS: $\quad(\mathrm{ESI})$

402.2 (100), 401.2 (20), 310.1 (8), 305.2 (21), 271.1(13)

HRMS: $\quad$ Calcd for $\mathrm{C}_{20} \mathrm{H}_{17} \mathrm{NONa}$ : 310.1208 , found 310.1219

TLC: $\quad R_{f} 0.14$ (hexanes/EtOAc, 4:1) [UV (254 nm)]

SFC: $\quad(2 R / S, 3 R / S)-6 \mathbf{b a}, \quad t_{\mathrm{R}} 9.8 \mathrm{~min}(1.3 \%) ;(2 S, 3 R)-6 \mathbf{b a}, t_{\mathrm{R}} 14.8 \mathrm{~min}(1.0 \%)$; $(2 R / S, 3 R / S)$-6ba, $t_{\mathrm{R}} 17.3 \mathrm{~min}(0.9 \%) ;(2 R, 3 S)$-6ba, $t_{\mathrm{R}} 23.8 \mathrm{~min}(96.8 \%)$ (Chiralpak OJ, $150 \mathrm{bar}, 40{ }^{\circ} \mathrm{C}, 2.75 \mathrm{~mL} / \mathrm{min}, 9 \% \mathrm{MeOH}$ in $\mathrm{CO}_{2}, 220 \mathrm{~nm}$ )

Opt. Rot.: $\quad[\alpha]_{\mathrm{D}}{ }^{24}-87\left(\mathrm{c}=0.52, \mathrm{CHCl}_{3}\right)$

Analysis: $\quad \mathrm{C}_{20} \mathrm{H}_{17} \mathrm{NO}(287.36)$

Calcd: $\quad$ C, 83.59; H, 5.96; $\quad$ N, $4.87 \%$

Found: $\quad$ C, $83.42 ; \quad \mathrm{H}, 5.83 ; \quad \mathrm{N}, 4.97 \%$

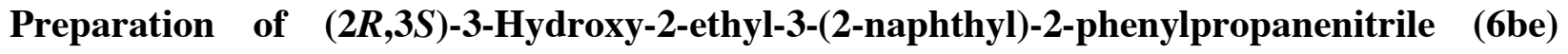
(Table 2, entry 2).

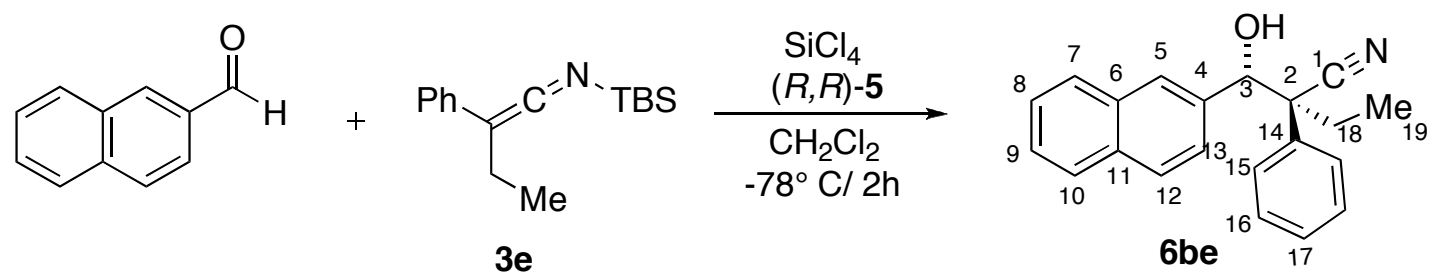

Following General Procedure 3, $156 \mathrm{mg}$ of 2-naphthaldehyde (1.0 mmol) was combined with $42 \mathrm{mg}$ of $(R, R)-5$ (0.05 mmol, 0.05 equiv), $126 \mu \mathrm{L}$ of $\mathrm{SiCl}_{4}(1.1 \mathrm{mmol}, 1.1$ equiv), and 0.94 $\mathrm{mL}$ of a $1.27 \mathrm{M}$ solution of silyl ketene imine $3 \mathbf{e}$ in $\mathrm{CH}_{2} \mathrm{Cl}_{2}(1.2 \mathrm{mmol}, 1.2$ equiv $)$ to yield after column chromatography $\left(\mathrm{SiO}_{2}(30 \mathrm{~g}), 3 \mathrm{~cm}\right.$ diam., hexanes/EtOAc, 4:1) $264 \mathrm{mg}$ of a white solid, 
that was recrystallized from hot EtOAc (ca. $0.5 \mathrm{~mL}$ ) and hexanes (ca. $1-0.5 \mathrm{~mL}$ ) to yield 234 $\mathrm{mg}(77 \%)$ of $6 \mathbf{b e}$ as white needles.

Data for 6be:

mp: $\quad 138-139^{\circ} \mathrm{C}$

가-NMR: $\quad\left(500 \mathrm{MHz}, \mathrm{CDCl}_{3}\right)$

7.85-7.78 (m, 4 H, HC(aryl)), 7.53-7.37 (m, 8 H, HC(aryl)), 5.05 (s, 1 H, HC(3)), $2.36(\mathrm{~s}, 1 \mathrm{H}, \mathrm{OH}), 2.08(\mathrm{dq}, J=7.2,7.2,1 \mathrm{H}, \mathrm{HC}(18)), 1.86(\mathrm{dq}, J=7.2,7.2,1 \mathrm{H}$, $\mathrm{HC}(18)), 0.82$ (dd, $\left.J=7.3,7.3,3 \mathrm{H}, \mathrm{H}_{3} \mathrm{C}(19)\right)$

${ }^{13} \mathrm{C}-\mathrm{NMR}: \quad\left(126 \mathrm{MHz}, \mathrm{CDCl}_{3}\right)$

135.8 (C(4)), 135.4 C(14)), 133.7 (C(11)), 132.9 (C(6)), 129.1 (C(15,16)), 128.5 (C(aryl)), 128.4 (C(17)), 128.0 (C(aryl)), 127.8 (C(aryl)), 127.5 (C(15,16)), 127.4 (C(aryl)), 127.6 (C(aryl)), 126.4 (C(aryl)), 125.2 (C(aryl)), 120.6 (C(1)), 80.0 (C(3)), 56.7 (C(2)), 28.8 (C(18)), 9.4 (C(19))

IR: $\quad(\mathrm{KBr}$ pellet $)$

3580 (s), 3056 (s), 2979 (s), 2930 (s), 2878 (s), 2237 (s), 1599 (s), 1506 (m), 1498 (s), 1466 (s), 1446 (s), 1380 (m), 1362 (s), 1272 (m), 1245 (m), 1193 (m), 1170 (s), 1123 (s), 1053 (s), 955 (s), 916 (s), 864 (s), 791(s), 757 (s), 669 (s)

MS: $\quad(\mathrm{ESI})$

325.1 (16), $324.1(100)$

HRMS: $\quad$ Calcd for $\mathrm{C}_{21} \mathrm{H}_{19} \mathrm{NONa:} 324.1364$, found 324.1364

TLC: $\quad R_{f} 0.17$ (hexanes/EtOAc, 4:1) [UV (254 nm)]

SFC: $\quad(2 R / R, 3 S / S)$-6be, $t_{\mathrm{R}} 11.4 \min (1.7 \%) ;(2 S, 3 R)-6 \mathbf{b e}, t_{\mathrm{R}} 13.3 \mathrm{~min}(1.2 \%)$; $(2 R / R, 3 S / S)$-6be, $t_{\mathrm{R}} 14.1 \min (1.3 \%)$; $(2 R, 3 S)$-6be, $t_{\mathrm{R}} 15.0 \mathrm{~min}(95.8 \%)$ (Chiralpak OD, 125 bar, $40{ }^{\circ} \mathrm{C}, 10 \% \mathrm{MeOH}$ in $\mathrm{CO}_{2}, 3.0 \mathrm{~mL} / \mathrm{min}, 220 \mathrm{~nm}$ )

Opt. Rot.: $\quad[\alpha]_{\mathrm{D}}^{24} 48.4\left(0.55, \mathrm{CHCl}_{3}\right)$

Analysis: $\quad \mathrm{C}_{21} \mathrm{H}_{19} \mathrm{NO}(301.38)$

$\begin{array}{llll}\text { Calcd: } & \text { C }, 83.69 ; & \text { H, 6.35; } & \text { N }, 4.65 \% \\ \text { Found: } & \text { C , 83.51; } & \text { H, 6.27; } & \text { N }, 4.73 \%\end{array}$




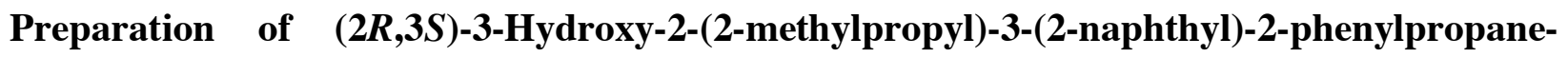
nitrile (6bf) (Table 2, entry 3)
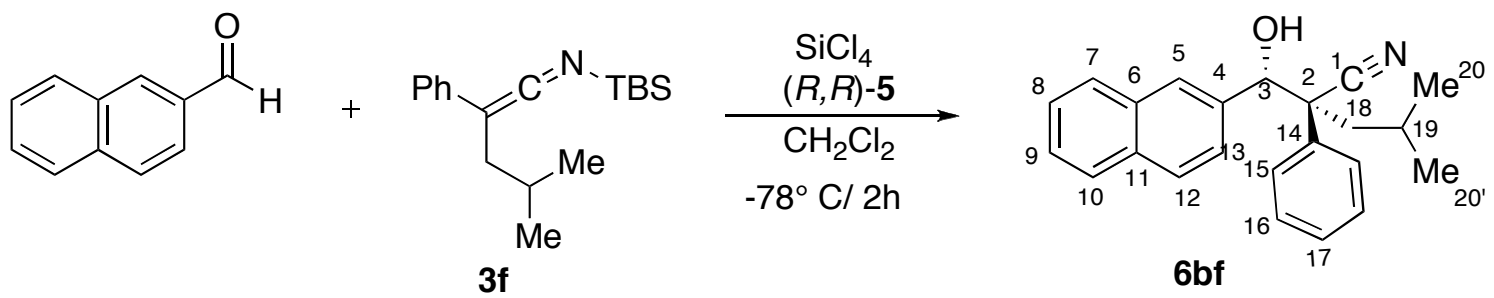

Following General Procedure 3, $156 \mathrm{mg}$ of 2-naphthaldehyde (1.0 mmol) was combined with $42 \mathrm{mg}$ of $(R, R)-5$ (0.05 mmol, 0.05 equiv), $126 \mu \mathrm{L}$ of $\mathrm{SiCl}_{4}(1.1 \mathrm{mmol}, 1.1$ equiv), and 0.75 $\mathrm{mL}$ of a $1.59 \mathrm{M}$ solution of silyl ketene imine $\mathbf{3 f}$ in $\mathrm{CH}_{2} \mathrm{Cl}_{2}$ (1.2 mmol, 1.2 equiv) to yield after column chromatography ( $\mathrm{SiO}_{2}(30 \mathrm{~g}), 3 \mathrm{~cm}$ diam., $\mathrm{CH}_{2} \mathrm{Cl}_{2} /$ hexanes, 7:1 to $\mathrm{CH}_{2} \mathrm{Cl}_{2} / \mathrm{EtOAc}, 7: 1$ ) $297 \mathrm{mg}(90 \%)$ of $\mathbf{6 b f}$ as a white, amorphous solid.

Data for $6 \mathbf{b f}$ :

mp: $\quad 154-156^{\circ} \mathrm{C}$

${ }^{1} \mathrm{H}-\mathrm{NMR}: \quad\left(500 \mathrm{MHz}, \mathrm{CDCl}_{3}\right)$

7.87-7.82 (m, 4 H, 4 x HC(aryl)), 7.56-7.49 (m, 5 H, 2 x HC(15), 3 x HC(aryl)), 7.46-7.38 (m, 3 H, 2 x HC(16), HC(17)), 5.00 (s, 1 H, HC(3)), 2.30 (br s, 1 H, OH), 2.07 (dd, $\left.J=14.1,5.0,1 \mathrm{H}, \mathrm{H}_{2} \mathrm{C}(18)\right), 1.64$ (dd, $J=14.2,7.8,1 \mathrm{H}, \mathrm{HC}(18)$ ), $1.54(\mathrm{~m}, 1 \mathrm{H}, \mathrm{HC}(19)), 0.88\left(\mathrm{~d}, J=6.4,3 \mathrm{H}, \mathrm{H}_{3} \mathrm{C}(20)\right), 0.66(\mathrm{~d}, J=6.6,3 \mathrm{H}$, $\left.\mathrm{H}_{3} \mathrm{C}\left(20^{\prime}\right)\right)$

${ }^{13} \mathrm{C}-\mathrm{NMR}: \quad\left(126 \mathrm{MHz}, \mathrm{CDCl}_{3}\right)$

135.9 (C(14)), 135.6 (C(4)), 133.7 (C(6,11)), 132.9 (C(6,11)), 129.1 (C(16)), 128.5 (C(17)), 128.0 (C(aryl)), 127.8 (C(aryl)), 127.6 (C(aryl)), 127.6 (C(15)), 126.6 (C(aryl)), 126.4 (C(aryl)), 125.4 (C(aryl)), 121.1 (C(1)), 81.0 (C(3)), 54.5 (C(2)), 43.6 (C(18)), 25.6 (C(19)), 24.2 (C(20')), 22.9 (C(20))

IR: $\quad(\mathrm{KBr}$ pellet $)$

3578 (s), 3058 (s), 3023 (m), 2958 (s), 2869 (s), 2236 (s), 1600 (s), 1507 (m), 1497 (s), 1469 (s), 1387 (m), 1364 (s), 1273 (m), 1243 (m), 1170 (s), 1123 (s), 1069 (s), 1045 (s), 1017 (s), 953 (m), 864 (s), 775 (s), 751 (s), 720 (s), 698 (s)

MS: $\quad(\mathrm{ESI})$

352.2 (10), $348.2(11), 347.2(65), 313.2(35), 312.2(100)$ 
HRMS: $\quad$ Calcd for $\mathrm{C}_{23} \mathrm{H}_{23} \mathrm{NONa:} 352.1688$, found 353.1677

TLC: $\quad R_{f} 0.21$ (hexanes/EtOAc, 4:1) [UV (254 nm)]

SFC: $\quad(2 R / R, 3 S / S)-6 \mathbf{b f}, t_{\mathrm{R}} 4.60 \min (1.0 \%)$; $(2 S, 3 R)-6 \mathbf{b f}, t_{\mathrm{R}} 5.1 \mathrm{~min}(0.4 \%)$; $(2 R / R, 3 S / S)-$ 6bf, $t_{\mathrm{R}} 5.6 \min (0.4 \%)$; $(2 R, 3 S)$-6bf, $t_{\mathrm{R}} 6.1 \mathrm{~min}(98.2 \%)$ (Chiralpak OD, 150 bar, $40{ }^{\circ} \mathrm{C}, 16 \% \mathrm{MeOH}$ in $\mathrm{CO}_{2}, 3.0 \mathrm{~mL} / \mathrm{min}, 220 \mathrm{~nm}$ )

Opt. Rot.: $\quad[\alpha]_{\mathrm{D}}{ }^{24}-107.1\left(\mathrm{c}=0.52, \mathrm{CHCl}_{3}\right)$

Analysis: $\quad \mathrm{C}_{23} \mathrm{H}_{23} \mathrm{NO}(329.43)$

$\begin{array}{llll}\text { Calcd: } & \text { C, 83.85; } & \text { H, 7.04; } & \text { N }, 4.25 \% \\ \text { Found: } & \text { C, 83.88; } & \text { H, 6.94; } & \text { N }, 4.44 \%\end{array}$

\section{Preparation of (2R,3S)-3-Hydroxy-2-(1-methylethyl)-3-(2-naphthyl)-2-phenylpropanenitrile (6bg) (Table 2, entry 4)}

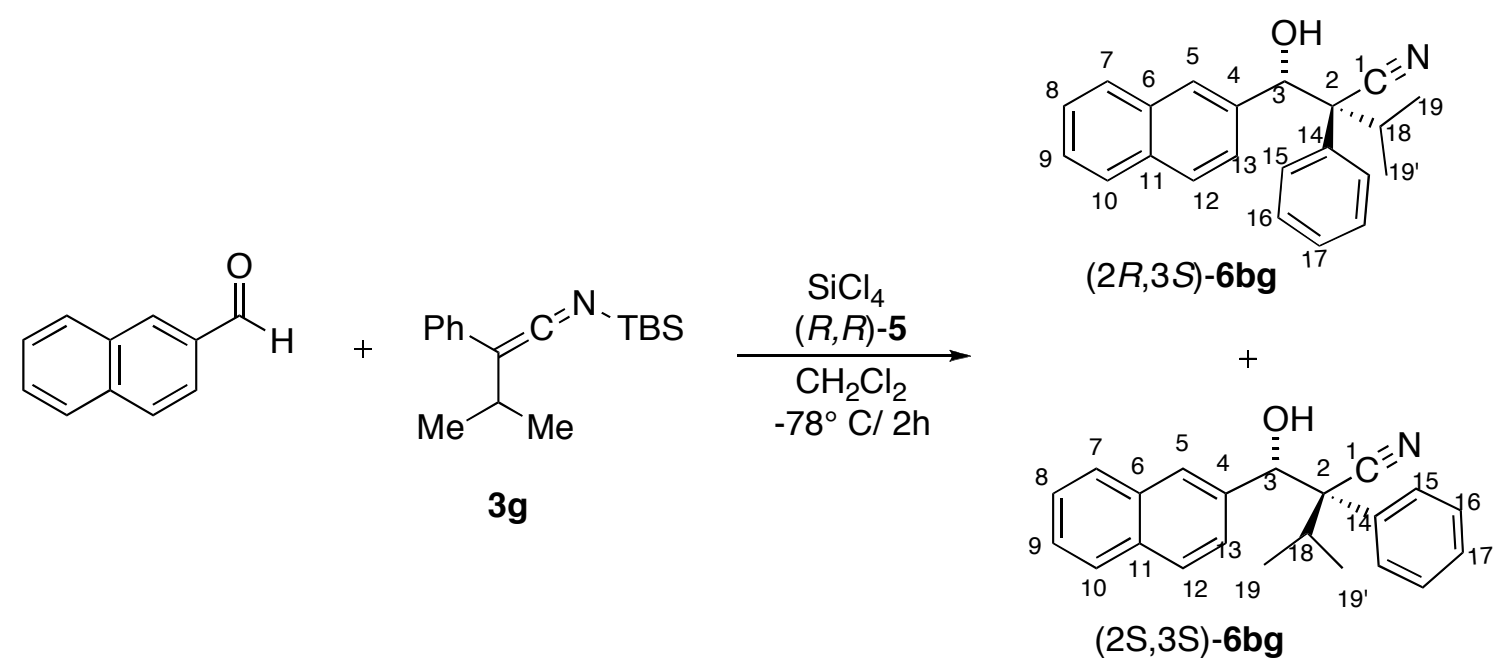

Following General Procedure 3, $156 \mathrm{mg}$ of 2-naphthaldehyde (1.0 mmol) was combined with $42 \mathrm{mg}$ of $(R, R)-5$ (0.05 mmol, 0.05 equiv), $126 \mu \mathrm{L}$ of $\mathrm{SiCl}_{4}(1.1 \mathrm{mmol}, 1.1$ equiv), and 0.71 $\mathrm{mL}$ of a $1.68 \mathrm{M}$ solution of silyl ketene imine $3 \mathrm{~g}$ in $\mathrm{CH}_{2} \mathrm{Cl}_{2}(1.2 \mathrm{mmol}, 1.2$ equiv) to yield after column chromatography $\left(\mathrm{SiO}_{2} \quad\left(\begin{array}{ll}30 & \mathrm{~g}\end{array}\right), 3 \mathrm{~cm}\right.$ diam., $\mathrm{CH}_{2} \mathrm{Cl}_{2} /$ hexanes, $6: 1$ to $\mathrm{CH}_{2} \mathrm{Cl}_{2} /$ hexanes/EtOAc, 6:1:1) $294 \mathrm{mg}$ of a white solid. The material was further purified by column chromatography $\left(\mathrm{SiO}_{2}(30 \mathrm{~g}), 3 \mathrm{~cm}\right.$ diam., toluene to toluene/EtOAc 9:1)) to yield $81 \mathrm{mg}$ $(26 \%)$ of $(2 S, 3 S)-6 \mathbf{b g}$ as a white foam and $149 \mathrm{mg}(47 \%)$ of $(2 R, 3 S)-\mathbf{6 b g}$, also as a white foam. The diastereo- and enantiomeric ratio was determined by SFC-analysis prior to separation of the diastereomers by further chromatography. 


\section{Data for $(2 R, 3 S)-\mathbf{6 b g}:^{8}$}

mp: $\quad 61-63^{\circ} \mathrm{C}$

마-NMR: $\quad\left(500 \mathrm{MHz}, \mathrm{CDCl}_{3}\right)$

7.76-7.74 (m, $1 \mathrm{H}, \mathrm{HC}(\operatorname{aryl}))$, 7.68-7.66 (m,1 H, HC(aryl)), 7.61 (d, J= 8.6, $1 \mathrm{H}$, HC(aryl)), 7.47-7.43 (m, 3 H, HC(aryl)), 7.23-7.17 (m, 3 H, HC(aryl)), 7.11-7.01 (m, 2 H, HC(aryl)), 7.06 (d, J = 8.6, 1 H, HC(aryl)), 5.47 (s, 1 H, HC(3)), 2.712.40 (m, 2 H, OH, HC(18)), 1.40 (d, $J=6.6,3$ H, $\mathrm{H}_{3} \mathrm{C}(19,19$ ')), 0.960 (d, $J=6.6$, $\left.3 \mathrm{H}, \mathrm{H}_{3} \mathrm{C}\left(19,19^{\prime}\right)\right)$

${ }^{13} \mathrm{C}-\mathrm{NMR}: \quad\left(126 \mathrm{MHz}, \mathrm{CDCl}_{3}\right)$ $136.4(\mathrm{C}(4)), 133.6$ (C(14)), 133.4 (C(11,6)), 132.7 (C(11,6)), $129.0(\mathrm{C}(15,16))$, 128.4 (C(aryl)), 128.2 (C(aryl)), $128.0 \quad(\mathrm{C}(15,16)), 127.7 \quad(\mathrm{C}(\operatorname{aryl})), 127.6$ (C(aryl)), 127.4 (C(aryl)), 126.6 (C(aryl)), 126.4 (C(aryl)), 125.4 (C(aryl)), 121.0 (C(1)), 76.2 (C(3)), $60.6(\mathrm{C}(2)), 33.3$ (C(18)), 19.8 (C(19, 19')), $19.2\left(\mathrm{C}\left(19,19^{\prime}\right)\right)$

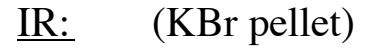

3446 (s), 3054 (s), 2967 (s), 2873 (s), 2237 (m), 1600 (m), 1495 (m), 1448 (m), 391 (s), 1369 (s), 1297 (m), 1271 (m), 1165 (m), 1137 (m), 1124(s), 1063 (s), 1033 (s), 953 (m), 894 (m), 859 (s), 820 (s), 748 (s), 711 (s)

MS: $\quad(E I, 70 \mathrm{eV})$ 315.0 (2.2), 159.0 (25.3), 158.0 (15.0), 157.0 (100.0), 156.0 (38.2), 155.0 (34.7), 144.0 (13.8), 129.0 (38.5), 128.0 (24.5), 127.0 (32.9), 117.0, (44.0), 90.0 (5.1), $89.0(6.3), 63.0(5.2)$

HRMS: (ESI)

Calcd for $\mathrm{C}_{22} \mathrm{H}_{21} \mathrm{NONa}$ : 338.1521, found 338.1531

TLC: $\quad 0.10 R_{f}\left(\mathrm{CH}_{2} \mathrm{Cl}_{2} /\right.$ hexanes, 6:1) [UV (254 nm)]

SFC: $\quad(2 S, 3 S)-6 \mathbf{b g}, t_{\mathrm{R}} 9.38 \mathrm{~min}(27.9 \%)$; $(2 S, 3 R)-6 \mathbf{b g}, t_{\mathrm{R}} 11.3 \min (12.9 \%)$; $(2 R, 3 R)-$ 6bg, $t_{\mathrm{R}} 12.5 \min (11.2 \%)$; (2R,3S)-6bg, $t_{\mathrm{R}} 13.8 \min (48.0 \%)$ (Chiralpak OD, 125 bar, $40{ }^{\circ} \mathrm{C}, 10 \% \mathrm{MeOH}$ in $\mathrm{CO}_{2}, 3.0 \mathrm{~mL} / \mathrm{min}, 220 \mathrm{~nm}$ )

Opt. Rot.: $\quad[\alpha]_{\mathrm{D}}^{24} 67.7\left(\mathrm{c}=0.50, \mathrm{CH}_{3} \mathrm{Cl}\right)$

Analysis: $\quad \mathrm{C}_{22} \mathrm{H}_{21} \mathrm{NO}(315.41)$

Calcd: $\quad$ C, 83.78; $\quad \mathrm{H}, 6.71 ; \quad \mathrm{N}, 4.44 \%$

Found: $\quad$ C, 83.43; H, 6.73; N, $4.50 \%$ 


\section{Data for $(2 S, 3 S)-\mathbf{6 b g}:^{8}$}

mp: $\quad 57-59^{\circ} \mathrm{C}$

${ }^{1} \mathrm{H}-\mathrm{NMR}: \quad\left(500 \mathrm{MHz}, \mathrm{CDCl}_{3}\right)$

7.75-737 (m, $1 \mathrm{H}, \mathrm{HC}(\operatorname{aryl}))$, 7.67-7.65 (m, $1 \mathrm{H}, \mathrm{HC}(\operatorname{aryl})), 7.57$ (d, J = 8.6, $1 \mathrm{H}$, HC(aryl)), 7.46-7.40 (m, 3 H, HC(aryl)), 7.30-7.21 (m, 5 H, HC(aryl)), 6.92 (dd, J = 8.6, 1.5, $1 \mathrm{H}, \mathrm{HC}(\operatorname{aryl})), 5.45(\mathrm{~s}, 1 \mathrm{H}, \mathrm{HC}(3)), 2.92(\mathrm{qq}, J=6.8,6.6,1 \mathrm{H}$, $\mathrm{HC}(18)), 2.18$ (br s, $1 \mathrm{H}, \mathrm{OH}), 1.41$ (d, $\left.J=6.6,3 \mathrm{H}, \mathrm{H}_{3} \mathrm{C}\left(19,19^{\prime}\right)\right), 0.90$ (d, $J=$ $\left.6.8,3 \mathrm{H}, \mathrm{H}_{3} \mathrm{C}\left(19,9^{\prime}\right)\right)$

${ }^{13} \mathrm{C}-\mathrm{NMR}: \quad\left(126 \mathrm{MHz}, \mathrm{CDCl}_{3}\right)$ 136.7 (C(4)), 133.9 (C(14)), 133.3 (C(11,6)), 132.6 (C(11,6)), $128.6(\mathrm{C}(15,16))$, 128.3 (C(aryl)), 128.2 (C(aryl)), $128.1 \quad(\mathrm{C}(15,16)), 127.6 \quad(\mathrm{C}(\operatorname{aryl})), 127.4$ (C(aryl)), 127.2 (C(aryl)), 126.4 (C(aryl)), 126.2 (C(aryl)), 125.0 (C(aryl)), 119.9 (C(1)), 75.9 (C(3)), 60.2 (C(2)), 32.3 (C(18)), 19.4 (C(19,19')), 18.6 (C(19,19'))

IR: $\quad(\mathrm{KBr}$ pellet $)$

3447 (s), 3054 (s), 2968 (s), 2930 (s), 2868 (m), 2237 (m), 1600 (m), 1507 (m), 1495 (m), 1468 (m), 1448 (m), 1390 (m), 1369 (m), 1271 (m), 1170 (s), 858 (s), $820(\mathrm{~s}), 747(\mathrm{~s}), 710(\mathrm{~s})$

MS: $\quad(E I, 70 \mathrm{eV})$ 315.0 (1.3), 159.0 (23.2), 158.0 (14.2), 157.0 (100.0), 156.0 (25.0), 155.0 (26.7), 144.0 (17.7), 129.0 (62.7), 128.0 (34.2), 127.0 (43.2), 117.0 (51.2), 116.0 (10.2), $75.0(7.8)$

HRMS: (ESI)

Calcd for $\mathrm{C}_{22} \mathrm{H}_{21} \mathrm{NONa:} 338.1521$, found 338.1528

TLC: $\quad R_{f} 0.20\left(\mathrm{CH}_{2} \mathrm{Cl}_{2} /\right.$ hexanes, 6/1) [UV (254 nm)]

SFC: $\quad(2 S, 3 S)-6 \mathbf{b g}, t_{\mathrm{R}} 9.38 \mathrm{~min}(27.9 \%) ;(2 S, 3 R)-\mathbf{6 b g}, t_{\mathrm{R}} 11.3 \mathrm{~min}(12.9 \%) ;(2 R, 3 S)-\mathbf{6 b g}$, $t_{\mathrm{R}} 12.5 \min (11.2 \%)$; (2R,3S)-6bg, $t_{\mathrm{R}} 13.8 \min (48.0 \%)$ (Chiralpak OD, 125 bar, $40{ }^{\circ} \mathrm{C}, 10 \% \mathrm{MeOH}$ in $\mathrm{CO}_{2}, 3.0 \mathrm{~mL} / \mathrm{min}, 220 \mathrm{~nm}$ )

Opt. Rot.: $\quad[\alpha]_{\mathrm{D}}{ }^{24} 96.4\left(\mathrm{c}=0.63, \mathrm{CHCl}_{3}\right)$

Analysis: $\quad \mathrm{C}_{22} \mathrm{H}_{21} \mathrm{NO}(315.41)$

Calcd: $\quad$ C, 83.78; $\quad$ H, 6.71; $\quad \mathrm{N}, 4.44 \%$

Found: $\quad$ C, 83.43; H, 6.73; N, $4.50 \%$ 
Preparation of $(2 R, 3 S)$-3-Hydroxy-2-(3-propenyl)-3-(2-naphthyl)-2-phenylpropanenitrile (6bh) (Table 2, entry 5)
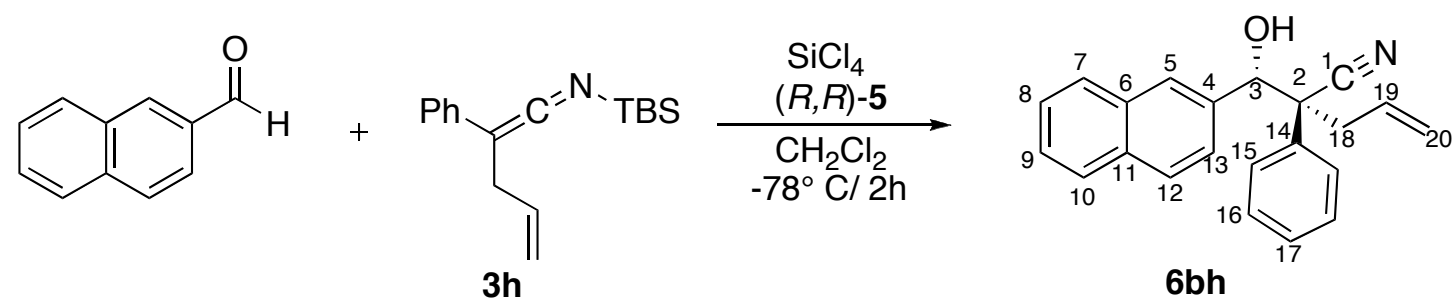

Following general Procedure 3,156 $\mathrm{mg}$ of 2-naphthaldehyde $(1.0 \mathrm{mmol})$ was combined with $42 \mathrm{mg}$ of $(R, R)-5$ ( $0.05 \mathrm{mmol}, 0.05$ equiv), $126 \mu \mathrm{L}$ of $\mathrm{SiCl}_{4}$ (1.1 mmol, 1.1 equiv), and 0.68 $\mathrm{mL}$ of a $1.76 \mathrm{M}$ solution of silyl ketene imine $3 \mathbf{h}$ in $\mathrm{CH}_{2} \mathrm{Cl}_{2}(1.2 \mathrm{mmol}, 1.2$ equiv) to yield after column chromatography $\left(\mathrm{SiO}_{2}(30 \mathrm{~g}), 3 \mathrm{~cm}\right.$ diam., $\mathrm{CH}_{2} \mathrm{Cl}_{2} /$ hexanes, 7:1 to $\left.\mathrm{CH}_{2} \mathrm{Cl}_{2} / \mathrm{EtOAc}, 7: 1\right)$ $264 \mathrm{mg}$ of a white solid, which was recrystallized from hot EtOAc (ca. $0.5 \mathrm{~mL}$ ) and hexanes (ca. $1-0.5 \mathrm{~mL})$ to yield $248 \mathrm{mg}(79 \%)$ of $\mathbf{6 b h}$ as white needles.

Data for $6 \mathbf{b h}$ :

mp: $\quad 138-139^{\circ} \mathrm{C}$

'H-NMR: $\quad\left(500 \mathrm{MHz}, \mathrm{CDCl}_{3}\right)$

7.85-7.78 (m, 2 H, HC(aryl)), 7.71 (s, $1 \mathrm{H}, \mathrm{HC}(5))$ ) 7.53-7.49 (m, $2 \mathrm{H}, \mathrm{HC}(\operatorname{aryl})$ ), 7.45-7.43 (m, 2 H, 1 x HC(15), 1 x HC(aryl)), 7.42-7.34 (m, 4 H, HC(aryl)), 5.56 (dddd, $J=17.2,10.2,7.1,7.1,1 \mathrm{H}, \mathrm{HC}(19)), 5.10$ (s, $1 \mathrm{H}, \mathrm{HC}(3)$ ), 5.13 (dd, $J=$ 17.4, 1.4, $\left.1 \mathrm{H}, \mathrm{H}_{2} \mathrm{C}(20)\right) 5.05$ (dd, $J=10.7,1.4,1 \mathrm{H}, \mathrm{H}_{2} \mathrm{C}(20)$ ), 2.85 (dd, $J=14.2$, 6.6, $\left.1 \mathrm{H}, \mathrm{H}_{2} \mathrm{C}(18)\right), 2.65$ (dd, $\left.J=14.2,7.6,1 \mathrm{H}, \mathrm{H}_{2} \mathrm{C}(18)\right), 2.51$ (br s, $1 \mathrm{H}, \mathrm{OH}$ )

${ }^{13} \mathrm{C}-\mathrm{NMR}: \quad\left(126 \mathrm{MHz}, \mathrm{CDCl}_{3}\right)$

135.5 (C(4)), 134.9 (C(14)), 133.6 (C(11)), 132.8 (C(6)), 131.5 (C(19)), 128.8 (C(16)), 128.5 (C(17)), 128.4 (C(13)), 127.9 (C(aryl)), 127.8 (C(15)), 127.8 (C(aryl)), $127.4(\mathrm{C}(5)), 126.6$ (C(aryl)), 126.4 (C(aryl)), 125.2 (C(13)), 120.5 (C(1)), $120.3(\mathrm{C}(20)), 79.3(\mathrm{C}(3)), 55.3(\mathrm{C}(2)), 39.6(\mathrm{C}(18))$

IR: $\quad(\mathrm{KBr}$ pellet $)$

3578 (s), 3058 (m), 2978 (m), 2930 (w), 2240 (m), 1600 (m), 1506 (m), 1498 (m), 1449 (m), 1359 (s), 1273 (m), 1249 (m), 1167 (m), 1122 (m), 1042 (s), 1018 (m), 983 (s), 922 (s), 867 (s), 829 (s), 779 (m), 755 (s), 726 (s), 696 (s) 
MS: $\quad(E S I)$

454.2 (12), 453.2 (50.0), 336.1 (9), 331.2 (15), 297.1 (32), 296.1 (100), 279.1

HRMS: $\quad$ Calcd for $\mathrm{C}_{22} \mathrm{H}_{19} \mathrm{NONa:} 336.1364$, found 336.1373

TLC: $\quad R_{f} 0.19$ (hexanes/EtOAc, 4:1) [UV (254 nm)]

SFC: $\quad(2 R, 3 S)-6 \mathbf{b h}, t_{\mathrm{R}} 17.2 \mathrm{~min}(91.7 \%)$; $(2 S, 3 R)-6 \mathbf{b h}, t_{\mathrm{R}} 21.7 \mathrm{~min}(2.3 \%) ;(2 R / R, 3 S / S)-$ 6bh, $t_{\mathrm{R}} 24.9 \min (6.0 \%)$ (Chiralpak AS, $150 \mathrm{bar}, 40{ }^{\circ} \mathrm{C}, 6.5 \% \mathrm{MeOH}$ in $\mathrm{CO}_{2}, 2.2$ $\mathrm{mL} / \mathrm{min}, 220 \mathrm{~nm}$ )

Opt. Rot.: $\quad[\alpha]_{\mathrm{D}}{ }^{24} 31.8\left(\mathrm{c}=0.5, \mathrm{CHCl}_{3}\right)$

Analysis: $\quad \mathrm{C}_{22} \mathrm{H}_{19} \mathrm{NO}(313.39)$

Calcd: $\quad$ C, 84.31; H, 6.11; N, $4.47 \%$

Found: $\quad$ C, 84.16; H, 5.96; $\quad$ N, $4.64 \%$

\section{Preparation of (3S)-2-Cyclohexaneyl-3-hydroxy-3-(2-naphthyl)propanenitrile (6bi) (Table} 2 , entry 6)<smiles>O=Cc1ccc2ccccc2c1</smiles>

$3 \mathbf{i}$

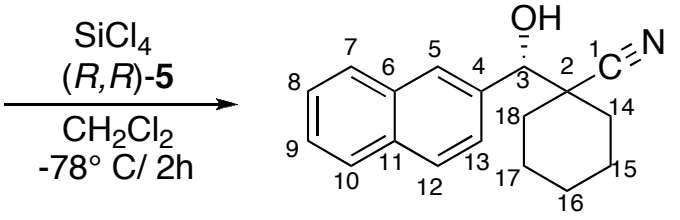

$6 \mathrm{bi}$

Following General Procedure 3, $156 \mathrm{mg}$ of 2-naphthaldehyde (1.0 mmol) was combined with $42 \mathrm{mg}$ of $(R, R)-5$ (0.05 mmol, 0.05 equiv), $126 \mu \mathrm{L}$ of $\mathrm{SiCl}_{4}$ (1.1 mmol, 1.1 equiv), and 0.65 $\mathrm{mL}$ of a $1.83 \mathrm{M}$ solution of silyl ketene imine $3 \mathbf{i}$ in $\mathrm{CH}_{2} \mathrm{Cl}_{2}$ (1.2 mmol, 1.2 equiv) to yield after column chromatography $\left(\mathrm{SiO}_{2}(30 \mathrm{~g}), 3 \mathrm{~cm}\right.$ diam., $\mathrm{CH}_{2} \mathrm{Cl}_{2} /$ hexanes, 9:1) $247 \mathrm{mg}$ of a white solid, which was recrystallized from hot EtOAc (ca. $0.5 \mathrm{~mL}$ ) and hexanes (ca. $1-0.5 \mathrm{~mL}$ ) to yield 224 $\mathrm{mg}(85 \%)$ of $\mathbf{6 b i}$ as white needles.

Data for 6bi:

mp: $\quad 176-178^{\circ} \mathrm{C}$

${ }^{1} \mathrm{H}-\mathrm{NMR}: \quad\left(500 \mathrm{MHz}, \mathrm{CDCl}_{3}\right)$

7.86-7.83 (m, 4 H, HC(5, 7, 10, 12)), 7.580 (dd, J = 8.4, 1.83, 1 H, HC(13)), 7.52-

7.49 (m, 2 H, HC(8, 9)), 4.66 (s, $1 \mathrm{H}, \mathrm{HC}(3)), 2.52$ (s, $1 \mathrm{H}, \mathrm{OH}), 2.33$ (dd, J = 
13.3, 1.5, $1 \mathrm{H}, \underline{\operatorname{H}} H C(14,18)), 1.79-1.75(\mathrm{~m}, 1 \mathrm{H}, \underline{\operatorname{HHC}}(15,17)), 1.72-1.58(\mathrm{~m}, 4$ $\mathrm{H}, \underline{\operatorname{H}} \mathrm{HC}(14,18)), 1.56-1.47$ (m, $1 \mathrm{H}, \underline{\operatorname{H}} \mathrm{HC}(15,17)), 1.43-1.37$ (m, $1 \mathrm{H}$, $\underline{\operatorname{HHC}}(14,18)), 1.33-1.28$ (m, 1 H, $\underline{H} H C(14,18)), 1.15-1.06$ (m, 1 H, HC(16))

${ }^{13} \mathrm{C}-\mathrm{NMR}: \quad\left(126 \mathrm{MHz}, \mathrm{CDCl}_{3}\right)$

136.6 (C(4)), 133.6 (C(11)), 132.9 (C(6)), 128.3 (C(12, 7, 10)), 128.2 (C(12, 7, 10)), 127.8 (C(12, 7, 10)), 126.9 (C(5)), 126.5 (C(8, 9)), $126.5(\mathrm{C}(8,9)), 125.1$ (C(13)), 122.1 (C(1)), $79.4(\mathrm{C}(3)), 46.1(\mathrm{C}(2)), 33.1$ (C(14, 18)), 31.8 (C(14, 18)), 25.3 (C(16)), $22.9(\mathrm{C}(15,17)), 22.7(\mathrm{C}(15,17))$

IR: $\quad(\mathrm{KBr}$ pellet $)$ 3435 (s), 3054 (m), 3023 (w), 2943 (s), 2888 (s), 2857 (s), 2242 (s), 1600 (m), 1509 (m), 1448 (s), 1367 (s), 1274 (m), 1243 (s), 1162 (m), 1124 (s), 1075 (s), 1048 (s), 958 (m), 936 (m), 912 (s), 872 (s), 824 (s), 778 (s), 760 (s)

MS: (ESI)

288.1 (10), 283.2 (15), 249.1 (13), 248.1 (100)

HRMS: $\quad$ Calcd for $\mathrm{C}_{18} \mathrm{H}_{19} \mathrm{NONa}$ : 288.1375, found 288.1364

TLC: $\quad R_{f} 0.16$ (hexanes/EtOAc, 4:1) [UV (254 nm)]

SFC: $\quad(3 R)-6 b i, t_{\mathrm{R}} 6.3 \min (8.7 \%)$; $(3 S)-6 b i, t_{\mathrm{R}} 8.2 \min (91.3 \%)$ (Chiralpak OD, 125 bar, $40{ }^{\circ} \mathrm{C}, 15 \% \mathrm{MeOH}$ in $\mathrm{CO}_{2}, 3.0 \mathrm{~mL} / \mathrm{min}, 220 \mathrm{~nm}$ )

Opt. Rot:: $\quad[\alpha]_{\mathrm{D}}^{24}-161.7\left(\mathrm{c}=0.52, \mathrm{CHCl}_{3}\right)$

Analysis: $\quad \mathrm{C}_{18} \mathrm{H}_{19} \mathrm{NO}(265.35)$

$\begin{array}{llll}\text { Calcd: } & \text { C }, 81.47 ; & \text { H, 7.22; } & \text { N, 5.28\% } \\ \text { Found: } & \text { C , 81.32; } & \text { H, 7.31; } & \text { N }, 5.41 \%\end{array}$


Preparation of (2R,3S)-3-Hydroxy-2-methyl-2-(1-methylethyl)-3-(2-naphthyl)propanenitrile (6bj) (Table 2, entry 7)
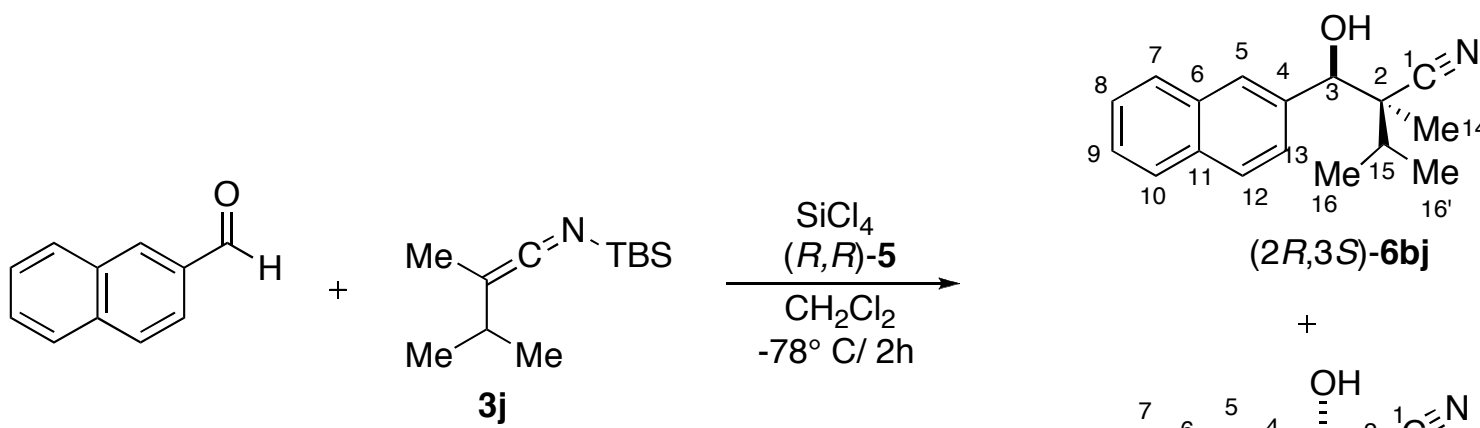

$(2 R, 3 S)-6 \mathbf{b j}$

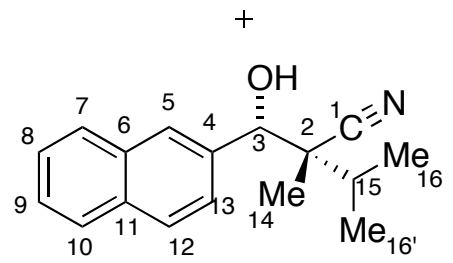

$(2 S, 3 S)-6 \mathbf{b j}$

Following General Procedure 3, $156 \mathrm{mg}$ of 2-naphthaldehyde (1.0 mmol) was combined with $42 \mathrm{mg}$ of $(R, R)-5$ (0.05 mmol, 0.05 equiv), $126 \mu \mathrm{L}$ of $\mathrm{SiCl}_{4}(1.1 \mathrm{mmol}, 1.1$ equiv), and 0.48 $\mathrm{mL}$ of a $2.5 \mathrm{M}$ solution of silyl ketene imine $\mathbf{3 j}$ in $\mathrm{CH}_{2} \mathrm{Cl}_{2}(1.2 \mathrm{mmol}, 1.2$ equiv) to yield after column chromatography $\left(\mathrm{SiO}_{2} \quad\left(\begin{array}{ll}30 & \mathrm{~g}\end{array}\right), \quad 3 \mathrm{~cm}\right.$ diam., $\mathrm{CH}_{2} \mathrm{Cl}_{2} /$ hexanes, 9:1 to $\mathrm{CH}_{2} \mathrm{Cl}_{2} /$ hexanes/EtOAc, 9:1:1) $254 \mathrm{mg}$ of a white solid. The material was further purified by column chromatography ( $\mathrm{SiO}_{2}(30 \mathrm{~g}), 3 \mathrm{~cm}$ diam., toluene to 9:1 toluene/EtOAc)) to yield 232 mg $(92 \%)$ of $\mathbf{6 b j}$ as an amorphous white powder. The diastereomeric mixture was separated by column chromatography $\left(\mathrm{SiO}_{2} \quad(30 \mathrm{~g}), \quad 3 \mathrm{~cm}\right.$ diam., $\mathrm{CH}_{2} \mathrm{Cl}_{2} /$ hexanes, 9:1 to $\mathrm{CH}_{2} \mathrm{Cl}_{2} /$ hexanes/EtOAc, 9:1:1) prior to characterization. ${ }^{9}$ The diastereo- and enantiomeric ratio was determined by SFC-analysis prior to separation of the diastereomers.

Data for $(2 R, 3 S)-\mathbf{6 b j}$ :

mp: $\quad 129-131^{\circ} \mathrm{C}$

${ }^{1} \mathrm{H}-\mathrm{NMR}: \quad\left(500 \mathrm{MHz}, \mathrm{CDCl}_{3}\right)$

7.92 (s, $1 \mathrm{H}, \mathrm{HC}(5)), 7.87-7.84$ (m, $3 \mathrm{H}, \mathrm{HC}(\operatorname{aryl})), 7.64$ (dd, $J$ = 8.6, 1.6, $1 \mathrm{H}$, HC(aryl)), 7.52-7.50 (m, $2 \mathrm{H}, \mathrm{HC}(\operatorname{aryl})), 4.98$ (s, 1H, HC(3)), 2.22 (br s, $1 \mathrm{H}$, $\mathrm{OH}), 1.94$ (qq, $J=6.8,6.8,1 \mathrm{H}, \mathrm{HC}(15)), 1.35$ (s, $\left.3 \mathrm{H}, \mathrm{H}_{3} \mathrm{C}(14)\right), 1.16$ (d, $J=6.8$, $\left.3 \mathrm{H}, \mathrm{H}_{3} \mathrm{C}\left(16,16^{\prime}\right)\right), 1.04\left(\mathrm{~d}, J=6.8,3 \mathrm{H}, \mathrm{H}_{3} \mathrm{C}\left(16,16^{\prime}\right)\right)$

${ }^{13} \mathrm{C}-\mathrm{NMR}: \quad\left(126 \mathrm{MHz}, \mathrm{CDCl}_{3}\right)$

$137.3(\mathrm{C}(4)), 133.5(\mathrm{C}(6)), 133.0(\mathrm{C}(7)), 128.3(\mathrm{C}(12,10,7)), 128.2$ 
(C(12,10, 7)), $127.8(\mathrm{C}(12,10,7)), 126.8(\mathrm{C}(5)), 126.6(\mathrm{C}(9,8)), 126.5(\mathrm{C}(9,8))$, 125.0 (C(13)), 122.2 (C(1)), $75.6(\mathrm{C}(3)), 47.9$ (C(2)), 31.9 (C(15)), 18.9 (C(16, $\left.\left.16^{\prime}\right)\right), 17.8\left(\mathrm{C}\left(16,16^{\prime}\right)\right), 16.3(\mathrm{C}(14))$

IR: $\quad(\mathrm{KBr}$ pellet $)$

3400 (s), 3059 (m), 2965 (s), 2925 (m), 2873 (s), 2253 (m), 1597 (m), 1508 (m), 1455 (m), 1376 (s), 1313 (m), 1273 (s), 1166 (s), 1125 (s), 1085 (s), 1047 (s), 954 (m), $892(\mathrm{~m}), 862(\mathrm{~s}), 826(\mathrm{~s}), 784(\mathrm{~s}), 754(\mathrm{~s}), 717(\mathrm{~m}), 651(\mathrm{~m})$

MS: $\quad(E I, 70 \mathrm{eV})$

253.1 (5.8), 158.0 (13.3), 157.0 (100.0), 155.0 (5.8), 130.1 (6.5), 129.0 (54.7), 128.0 (17.8), $127.0(13.9)$

HRMS: $\quad$ Calcd for $\mathrm{C}_{17} \mathrm{H}_{19} \mathrm{NO}: 253.1467$, found 253.1467

TLC: $\quad R_{f} 0.10\left(\mathrm{CH}_{2} \mathrm{Cl}_{2} /\right.$ hexanes, $\left.6: 1\right)$ [UV $(254 \mathrm{~nm})$ ]

SFC: $\quad(2 S, 3 R)-6 \mathbf{b j}, t_{\mathrm{R}} 12.1 \mathrm{~min}(4.7 \%) ;(2 R, 3 S)-6 \mathbf{b j}, t_{\mathrm{R}} 14.2 \mathrm{~min}(54.7 \%) ;(2 R / S, 3 R / S)-$ 6bj, $t_{\mathrm{R}} 18.9 \min (40.6 \%)$ (Chiralpak AS, 150 bar, $40{ }^{\circ} \mathrm{C}, 4 \% \mathrm{MeOH}$ in $\mathrm{CO}_{2}, 2.5$ $\mathrm{mL} / \mathrm{min}, 220 \mathrm{~nm}$ )

Opt. Rot.: $\quad[\alpha]_{D}^{24} 58.9(\mathrm{c}=0.54)$

Analysis: $\quad \mathrm{C}_{18} \mathrm{H}_{19} \mathrm{NO}(253.34)$

$\begin{array}{llll}\text { Calcd: } & \text { C, 80.60; } & \text { H, 7.56; } & \text { N, 5.53\% } \\ \text { Found: } & \text { C, 80.87; } & \text { H, 7.43; } & \text { N, 5.66\% }\end{array}$

Data for $(2 S, 3 S)-\mathbf{6} \mathbf{b} \mathbf{j}:{ }^{8}$

mp: $\quad 128-133{ }^{\circ} \mathrm{C}$

1H-NMR: $\quad\left(500 \mathrm{MHz}, \mathrm{CDCl}_{3}\right)$

7.87-7.84 (m, $4 \mathrm{H}, \mathrm{HC}(5,12,10,7)), 7.61$ (dd, $J=8.6,1.4,1 \mathrm{H}, \mathrm{HC}(13))$, 7.52-7.50 (m, $2 \mathrm{H}, \mathrm{HC}(8,9)$ ), 4.89 (s, $1 \mathrm{H}, \mathrm{HC}(3)$ ), 2.46 (br s, $1 \mathrm{H}, \mathrm{OH}$ ), 2.29 (qq, $J=6.8$, 6.8, $1 \mathrm{H}, \mathrm{HC}(15)), 1.24\left(\mathrm{~d}, J=6.8,3 \mathrm{H}, \mathrm{H}_{3} \mathrm{C}\left(16,16^{\prime}\right)\right), 1.05(\mathrm{~d}, J=6.6,3 \mathrm{H}$, $\left.\mathrm{H}_{3} \mathrm{C}\left(16,166^{\prime}\right)\right), 1.04$ (s, $\left.3 \mathrm{H}, \mathrm{H}_{3} \mathrm{C}(14)\right)$

${ }^{13} \mathrm{C}-\mathrm{NMR}: \quad\left(126 \mathrm{MHz}, \mathrm{CDCl}_{3}\right)$

$136.9(\mathrm{C}(4)), 133.6(\mathrm{C}(6)), 132.9(\mathrm{C}(7)), 128.3(\mathrm{C}(12,10,7)), 128.3(\mathrm{C}(12,10,7))$, $127.8(\mathrm{C}(12,10,7)), 127.1(\mathrm{C}(5)), 126.6(\mathrm{C}(9,8)), 126.5(\mathrm{C}(9,8)), 125.1(\mathrm{C}(13))$, $122.9(\mathrm{C}(1)), 75.5(\mathrm{C}(3)), 48.1(\mathrm{C}(2)), 31.6(\mathrm{C}(15)), 19.3(\mathrm{C}(16,16)), 16.7(\mathrm{C}(16$, 
16 ')), $15.8(\mathrm{C}(14))$

IR: $\quad(\mathrm{KBr}$ pellet $)$

3549 (s), 2965 (s), 2935 (m), 2904 (m), 2878 (m), 2240 (m), 1505 (m), 1463 (m), 1395 (m), 1376 (s), 1306 (m), 1270 (s), 1166 (m), 1124 (m), 1057 (s), 898 (s),

$869(\mathrm{~s}), 832(\mathrm{~s}), 775(\mathrm{~m}), 761$ (s)

MS: $\quad(\mathrm{EI}, 70 \mathrm{eV})$

253.1 (2.4), 158.0 (13.1), 157.0 (100.0), 155.0 (9.2), 129.0 (77.7), 128.0 (32.3), 127.0 (25.9), 77.0 (8.5), 75.0 (12.9), 73.0 (10.9)

HRMS: $\quad$ Calcd for $\mathrm{C}_{17} \mathrm{H}_{19} \mathrm{NO}: 253.1467$, found 253.1468

TLC: $\quad R_{f} 0.07\left(\mathrm{CH}_{2} \mathrm{Cl}_{2} /\right.$ hexanes, 6:1) [UV (254 nm)]

SFC: $\quad(2 R / S, 3 R / S)-6 \mathbf{b j}, t_{\mathrm{R}} 6.53 \min (59.3 \%)$; (2S,3S)-6bj, $t_{\mathrm{R}} 7.09 \min (39.4 \%) ;(2 R, 3 R)-$ 6bj, $t_{\mathrm{R}} 8.0 \min \left(1.3 \%\right.$ ) (Chiralpak AD, 125 bar, $40{ }^{\circ} \mathrm{C}, 8 \% \mathrm{MeOH}$ in $\mathrm{CO}_{2}, 3.0$ $\mathrm{mL} / \mathrm{min}, 220 \mathrm{~nm}$ )

Opt. Rot.: $\quad[\alpha]_{\mathrm{D}}{ }^{24} 51.8\left(\mathrm{c}=0.51, \mathrm{CHCl}_{3}\right)$

Analysis: $\quad \mathrm{C}_{18} \mathrm{H}_{19} \mathrm{NO}(253.34)$

Calcd: $\quad$ C, 80.60; H, 7.56; N, 5.53\%

Found: $\quad$ C, $80.87 ; \quad H, 7.43 ; \quad \quad N, 5.66 \%$

\section{Addition of Silyl Ketene Imine 3a to Different Aldehydes.}

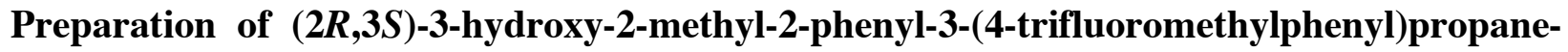
nitrile (6ca) (Table 3, entry 1)

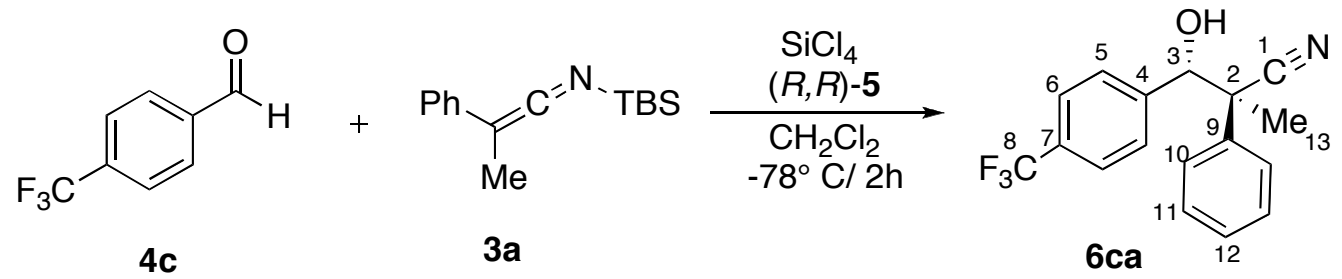

Following General Procedure 2, $174.5 \mathrm{mg}$ of 4-(trifluoromethyl)benzaldehyde (1.0 mmol, 1.0 equiv) was combined with $42 \mathrm{mg}$ of $(R, R)-5$ (0.05 mmol, 0.05 equiv), $126 \mu \mathrm{L}$ of $\mathrm{SiCl}_{4}(1.1$ mmol, 1.1 equiv), and $0.86 \mathrm{~mL}$ of a $1.4 \mathrm{M}$ solution of silyl ketene imine 3a (1.2 mmol, 1.2 equiv, $1.4 \mathrm{M}$ soln. in $\left.\mathrm{CH}_{2} \mathrm{Cl}_{2}\right)$ to yield after column chromatography $\left(\mathrm{SiO}_{2}(30 \mathrm{~g}) 3 \mathrm{~cm}\right.$ diam. hexanes/EtOAc, 4:1) $292 \mathrm{mg}(95 \%)$ of $\mathbf{6 c a}$ as a white solid, which was crystallized from $0.1 \mathrm{~mL}$ 
hot toluene and hexanes (ca. $0.5 \mathrm{~mL}$ ) to yield $269 \mathrm{mg}(88 \%)$ of white needles.

\section{Data for 6ca:}

mp: $\quad 125-126^{\circ} \mathrm{C}$

${ }^{1} \mathrm{H}$ NMR: $\quad\left(500 \mathrm{MHz}, \mathrm{CDCl}_{3}\right)$

7.60-7.56 (m, $2 \mathrm{H}, \mathrm{HC}(6))$, 7.47-7.37 (m, $7 \mathrm{H}, \mathrm{HC}(5,10,11,12)), 4.95$ (s, $1 \mathrm{H}$, $\mathrm{HC}(3)), 1.62$ (s, $\left.3 \mathrm{H}, \mathrm{H}_{3} \mathrm{C}(13)\right)$

${ }^{13} \mathrm{C} \mathrm{NMR:} \quad\left(125 \mathrm{MHz}, \mathrm{CDCl}_{3}\right)$

$141.6(\mathrm{C}(4,9)), 136.7(\mathrm{C}(4,9)), 130.8(\mathrm{q}, J=32.2, \mathrm{C}(7)), 128.9(\mathrm{C}(11)), 128.6$ (C(12)), $128.0(\mathrm{C}(5)), 126.7$ (C(10)), 124.9 (q, $J=3.7, \mathrm{C}(6)), 122.8$ (q, $J=272.1$, C(8)), 121.5 (C(1)), 79.0 (C(3)), 49.3 (C(2)), 22.0 (C(13))

IR: $(\mathrm{KBr}$ pellet)

3434 (m), 3073 (w), 3030 (w), 2999 (w), 2909 (w), 2247 (w), 1623 (w), $1601(\mathrm{w})$, 1448 (w), 1420 (w), 1332 (m), 1163 (m), 1130 (m), 1111 (m), 1072 (m), 1050 (m), $1019(\mathrm{~m}), 842(\mathrm{~m}), 763(\mathrm{w}), 743(\mathrm{w}), 711(\mathrm{~m}), 695(\mathrm{~m}), 636(\mathrm{w})$

MS: (ESI)

132 (66), 156 (14), 279 (18), 186 (90), 288 (22), 323 (16), $328\left(\mathrm{M}^{+}+\mathrm{Na}, 100\right), 411$ (14), 419 (10)

HRMS Calcd for $\mathrm{C}_{17} \mathrm{H}_{14} \mathrm{~F}_{3} \mathrm{NONa}$ : 328.0925 , found: 328.0933

Opt. Rot.: $[\alpha]_{\mathrm{D}}{ }^{24} 12.9\left(\mathrm{c}=0.51, \mathrm{CHCl}_{3}\right)$

TLC: $\quad R_{f} 0.25$ (hexanes/EtOAc, 4:1) [UV (254 nm)]

SFC: $\quad(2 R, 3 S)-6 \mathbf{c a}, t_{\mathrm{R}} 7.67 \mathrm{~min},(99.1 \%) ;(R, R) /(S, S)-\mathbf{6 c a}, t_{\mathrm{R}} 8.13 \min (0.22 \%) ;(2 S, 3 R)-$ 6ca, $t_{\mathrm{R}} 9.26 \min (0.68 \%)$ (Chiralpak AD, 125 bar, $40^{\circ} \mathrm{C}, 4 \% \mathrm{MeOH}$ in $\mathrm{CO}_{2} 2.5$ $\mathrm{mL} / \mathrm{min}$ )

Analysis: $\mathrm{C}_{17} \mathrm{H}_{14} \mathrm{~F}_{3} \mathrm{NO}(305.29)$

Calcd: C, 66.88; H, 4.62; N, 4.59\%

Found: $\quad$ C, 66.82; $\quad \mathrm{H}, 4.57 ; \quad \mathrm{N}, 4.67 \%$ 
Preparation of (2R,3S)-3-Hydroxy-2-methyl-2-phenyl-3-(4-bromophenyl)propanenitrile (6da) (Table 3, entry 2)<smiles>O=Cc1ccc(Br)cc1</smiles>

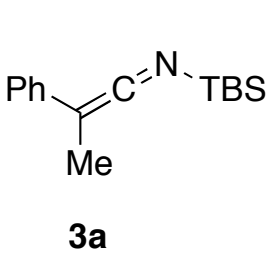
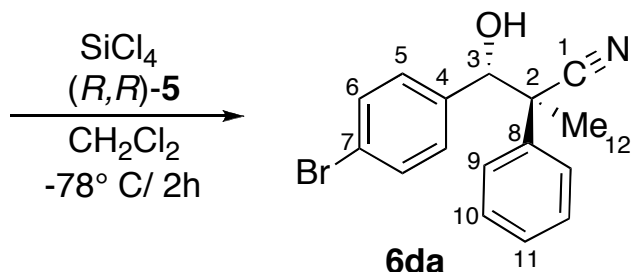

Following General Procedure 2, $185 \mathrm{mg}$ of 4-bromobenzaldhyde (1.0 mmol) was combined with $42 \mathrm{mg}$ of $(R, R)-5$ (0.05 mmol, 0.05 equiv), $126 \mu \mathrm{L}$ of $\mathrm{SiCl}_{4}(1.1 \mathrm{mmol}, 1.1$ equiv), and $0.95 \mathrm{~mL}$ of a $1.25 \mathrm{M}$ solution of silyl ketene imine $3 \mathbf{a}$ in $\mathrm{CH}_{2} \mathrm{Cl}_{2}$ (1.2 mmol, 1.2 equiv) to yield after column chromatography $\left(\mathrm{SiO}_{2}(30 \mathrm{~g}), 3 \mathrm{~cm}\right.$ diam., hexanes/EtOAc, 4:1) 303 $\mathrm{mg}$ of a white solid, which was recrystallized from hot benzene (ca. $0.5 \mathrm{~mL}$ ) and pentanes (ca. 1 - $0.5 \mathrm{~mL})$ to yield $294 \mathrm{mg}(93 \%)$ of $\mathbf{6 d a}$ as white needles.

Data for 6da:

mp: $109-110^{\circ} \mathrm{C}$

${ }^{1} \mathrm{H}$ NMR: $\quad\left(500 \mathrm{MHz}, \mathrm{CDCl}_{3}\right)$

7.45-7.37 (m, $7 \mathrm{H}, \mathrm{HC}(6,9,10,11)), 7.13$ (d, $J=8.5,2 \mathrm{H}, \mathrm{HC}(5)), 4.84$ (s, $1 \mathrm{H}$, $\mathrm{HC}(3)), 2.44$ (s, $1 \mathrm{H}, \mathrm{OH}), 1.60$ (s, $\left.3 \mathrm{H}, \mathrm{H}_{3} \mathrm{C}(12)\right)$

${ }^{13} \mathrm{C} \mathrm{NMR:} \quad\left(125 \mathrm{MHz}, \mathrm{CDCl}_{3}\right)$

$137.0(\mathrm{C}(4,8)), 136.8(\mathrm{C}(4,8)), 131.3(\mathrm{C}(9,10,6)), 129.4(\mathrm{C}(6)), 129.1(\mathrm{C}(9,10$, 6)), $128.7(\mathrm{C}(11)), 126.9(\mathrm{C}(9,10,6)), 123.1(\mathrm{C}(7)), 121.7(\mathrm{C}(1)), 79.3(\mathrm{C}(3)), 49.5$ $(\mathrm{C}(2)), 22.3(\mathrm{C}(12))$

IR: $(\mathrm{KBr}$ pellet $)$

3430 (s), 3054 (w), 3023 (s), 2992 (m), 2945 (m), 2894 (m), 2242 (m), 1490 (s), 1446 (s), 1404 (s), 1302 (m), 1238 (m), 1199 (m), 1073 (s), 1046 (s), 1010 (s), 827 (s), 737(s), $696(\mathrm{~s})$

MS: (ESI)

466.2 (24), 449.0 (18), 431.1 (41), 429.1 (37), 411.3 (18), 340.0 (100), 338.0 (92), 334.1 (22), 298.0 (56), 133.1 (48), 130.2 (45)

$\underline{\text { HRMS }}$ Calcd for $\mathrm{C}_{16} \mathrm{H}_{14} \mathrm{BrNONa}, 338.0155$, found: 338.0163 
Opt. Rot.: $[\alpha]_{\mathrm{D}}^{24} 16\left(\mathrm{c}=0.46, \mathrm{CHCl}_{3}\right)$

TLC: $\quad R_{f} 0.16$ (hexanes/EtOAc, 4:1) [UV (254 nm]

SFC: $\quad(2 S, 3 R)-\mathbf{6 d a}, t_{\mathrm{R}} 8.68 \mathrm{~min}(1.0 \%)$; $(2 R / S, 3 R / S)-6 \mathbf{d a}, t_{\mathrm{R}} 10.1 \mathrm{~min}(0.6 \%) ;(2 R / S, 3 R / S)-$ 6da, $t_{\mathrm{R}} 11.2 \min (0.5 \%)$; (2R,3S)-6da, $t_{\mathrm{R}} 13.6 \min (97.9 \%)$ (Chiralpak OB, 125 bar, $40{ }^{\circ} \mathrm{C}, 5 \% \mathrm{MeOH}$ in $\mathrm{CO}_{2}, 2.5 \mathrm{~mL} / \mathrm{min}, 220 \mathrm{~nm}$ )

Analysis: $\mathrm{C}_{16} \mathrm{H}_{14} \mathrm{BrNO}$ (316.19)

Calcd: $\quad$ C, $60.78 ; \quad \mathrm{H}, 4.46 ; \quad \mathrm{N}, 4.43 \%$

Found: $\quad$ C, $60.95 ; \quad H, 4.35 ; \quad$ N, $4.33 \%$

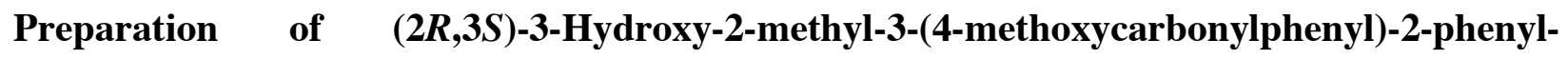
propanenitrile (6ea) (Table 3 , entry 3 )<smiles>COC(=O)c1ccc(C(=O)I)cc1</smiles>

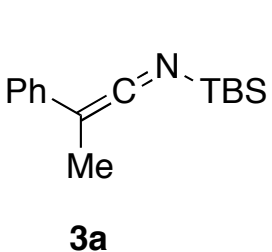

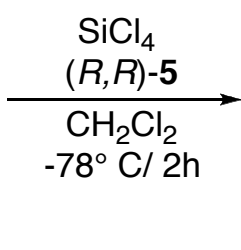<smiles>COC(=O)c1ccc(C(O)C(C#N)(C#N)c2ccccc2)cc1</smiles>

Following General Procedure 2, $164 \mathrm{mg}$ of methyl 4-formylbenzoate (1.0 mmol, 1.0 equiv) was combined with $42 \mathrm{mg}$ of $(R, R)-5$ (0.05 mmol, 0.05 equiv), $126 \mu \mathrm{L}$ of $\mathrm{SiCl}_{4}(1.1$ mmol, 1.1 equiv), and $0.86 \mathrm{~mL}$ of a $1.4 \mathrm{M}$ solution of silyl ketene imine 3a (1.2 mmol, 1.2 equiv, $1.4 \mathrm{M}$ in $\left.\mathrm{CH}_{2} \mathrm{Cl}_{2}\right)$ to yield after column chromatography $\left(\mathrm{SiO}_{2}(30 \mathrm{~g}) 3 \mathrm{~cm}\right.$ diam. EtOAc/hexanes 1/4) $289 \mathrm{mg}(97 \%)$ of 6ea as a white solid, which was crystallized from hot EtOAc (0.5 mL) and hexanes $(0.5 \mathrm{~mL})$ to yield $277 \mathrm{mg}(93 \%)$ of $\mathbf{6 e a}$ as white plates.

Data for 6ea:

mp: $\quad 112-114^{\circ} \mathrm{C}$

$1{ }^{1 \mathrm{H}} \mathrm{NMR}: \quad\left(500 \mathrm{MHz}, \mathrm{CDCl}_{3}\right)$

$7.92(\mathrm{~d}, J=8.4,2 \mathrm{H}, \mathrm{HC}(6)), 7.44-7.33(\mathrm{~m}, 5 \mathrm{H}, \mathrm{HC}(11,12,13)), 7.28$ (d, $J=8.3,2$ H, HC(5)), 4.94 (s, $1 \mathrm{H}, \mathrm{HC}(3)), 3.88$ (s, $\left.3 \mathrm{H}, \mathrm{H}_{3} \mathrm{C}(9)\right), 2.77$ (br s, $1 \mathrm{H}, \mathrm{OH}$ ), 1.60 (s, $\left.3 \mathrm{H}, \mathrm{H}_{3} \mathrm{C}(14)\right)$

${ }^{13} \mathrm{C}$ NMR: $\quad\left(125 \mathrm{MHz}, \mathrm{CDCl}_{3}\right)$

$166.8(\mathrm{C}(8)), 142.7(\mathrm{C}(4,10)), 136.8(\mathrm{C}(4,10)), 130.2$ (C(7)), $129.1(\mathrm{C}(6)), 128.8$ 
(C(12)), 128.5 (C(13)), 127.6 (C(5)), 126.75 (C(11)), $121.7(\mathrm{C}(1)), 79.1(\mathrm{C}(3)), 52.2$

(C(9)), $49.2(\mathrm{C}(2)), 21.9(\mathrm{C}(14))$

IR: $(\mathrm{KBr}$ pellet)

3493 (m), 3419 (s), 3063 (m), 3036 (m), 3003 (m), 2951 (m), 2884 (m), 1718 (s), 1698 (s), 1612 (m), 1493 (m), 1446 (s), 1436 (s), 1418 (s), 1381 (m), 1281 (s), 1189

(m), 1115 (s), 1019 (s), 959 (m), 889 (m), 853 (m), 783 (m), 728 (s), 697 (s), 640

$(\mathrm{m})$

MS: (ESI)

$296\left(\mathrm{M}^{+}+\mathrm{H}, 87\right), 264(9), 165(21), 133(9), 132(100)$

$\underline{\text { HRMS }}$ Calcd for $\mathrm{C}_{18} \mathrm{H}_{18} \mathrm{NO}_{3}: 296.1287$, found: 296.1299

Opt. Rot.: $[\alpha]_{\mathrm{D}}^{24} 5.5\left(\mathrm{c}=0.52, \mathrm{CHCl}_{3}\right)$

TLC: $\quad R_{f} 0.11$ (hexanes/EtOAc, 4:1) [UV (254 nm)]

SFC: $\quad(2 S, 3 R)$-6ea, $t_{\mathrm{R}} 5.20 \min (1.4 \%) ;(R, R) /(S, S)$-6ea, $t_{\mathrm{R}} 5.80 \mathrm{~min}(0.8 \%) ;(2 R, 3 S)$-6ea, $t_{\mathrm{R}}$ $7.60 \mathrm{~min}(97.8 \%)$ (Chiralpak OB, $120 \mathrm{bar}, 40^{\circ} \mathrm{C}, 10 \% \mathrm{MeOH}$ in $\mathrm{CO}_{2}, 2 \mathrm{~mL} / \mathrm{min}$ )

Analysis: $\mathrm{C}_{18} \mathrm{H}_{17} \mathrm{NO}_{3}(295.33)$

Calcd: C, 73.19; H, 5.80; N, 4.74\%

Found: C, 73.25; H, 5.84; N, $4.90 \%$

\section{Preparation of (2R,3S)-3-Hydroxy-3-(4-methoxyphenyl)-2-methyl-2-phenylpropanenitrile} (6fa) (Table 3, entry 4)

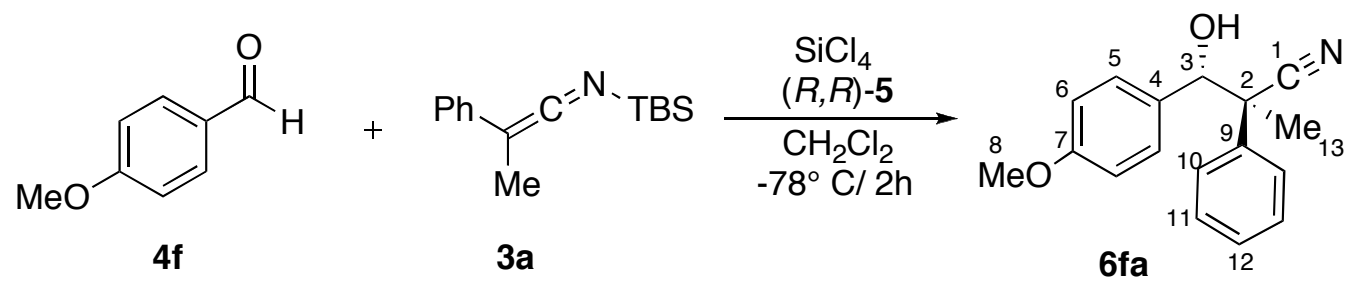

Following General Procedure 2, $136.2 \mathrm{mg}$ of 4-methoxybenzaldehyde (1.0 mmol, 1.0 equiv) was combined with $42 \mathrm{mg}$ of $(R, R)-5$ (0.05 mmol, 0.05 equiv), $126 \mu \mathrm{L}$ of $\mathrm{SiCl}_{4}(1.1$ mmol, 1.1 equiv), and $0.86 \mathrm{~mL}$ of a $1.4 \mathrm{M}$ solution of silyl ketene imine $3 \mathbf{a}$ (1.2 mmol, 1.2 equiv) to yield after column chromatography $\left(\mathrm{SiO}_{2}(27 \mathrm{~g}) 3 \mathrm{~cm}\right.$ diam. EtOAc/hexanes 1/4 ) 227 $\mathrm{mg}(85 \%)$ of $\mathbf{6 f a}$ as a white solid, which was crystallized from boiling $\mathrm{Et}_{2} \mathrm{O}(2 \mathrm{~mL})$ and hexanes 
(4 mL) to yield $210 \mathrm{mg}(78 \%)$ of $\mathbf{6 f a}$ as white needles.

Data for 6fa:

mp: $117-120^{\circ} \mathrm{C}$

${ }^{1} \mathrm{H}$ NMR: $\quad\left(500 \mathrm{MHz}, \mathrm{CDCl}_{3}\right)$

7.49-7.45 (m, 2 H, HC(10)), 7.43-7.34 (m, 3 H, HC(11,12)), 7.24-7.20 (m, 2 H, $\mathrm{HC}(5))$, 6.87-6.83 (m, $2 \mathrm{H}, 2 \mathrm{H}, \mathrm{HC}(6)), 4.81$ (s, $1 \mathrm{H}, \mathrm{HC}(3)), 3.81$ (s, $3 \mathrm{H}$, $\mathrm{H}_{3} \mathrm{C}(8)$ ), 2.38 (br s, $\left.1 \mathrm{H}, \mathrm{OH}\right), 1.58$ (s, $3 \mathrm{H}, \mathrm{H}_{3} \mathrm{C}(13)$ )

${ }^{13} \mathrm{C} \mathrm{NMR:} \quad\left(125 \mathrm{MHz}, \mathrm{CDCl}_{3}\right)$

159.8 (C(7)), 137.6 (C(9)), 129.9 (C(4)), 128.7 (C(5,11)), 128.7 (C(5,11)), 128.2 (C(12)), 126.7 (C(10)), 121.9 (C(1)), 113.4 (C(6)), 79.4 (C(3)), 55.2 (C(8)), 49.5 (C(2)), $22.6(\mathrm{C}(13))$

IR: $(\mathrm{KBr}$ pellet)

3468 (s), 3060 (s), 3033 (s), 3011 (m), 3001 (m), 2973 (m), 2940 (m), 2246 (m), 1611 (s), 1598 (m), 1513 (s), 1459 (s), 1446 (s), 1286 (s), 1244 (s), 1176 (s), 1072 (s), 1040 (s), 1020 (s), 898 (m), 832 (s), 758 (s), 747 (s), 693 (s), 658 (m)

MS: (ESI)

250 (100), 251 (17), 285 (29), 290 (38)

$\underline{\text { HRMS }}$ Calcd for $\mathrm{C}_{17} \mathrm{H}_{17} \mathrm{NO}_{2} \mathrm{Na}: 290.1157$, found: 290.1149

Opt. Rot.: $[\alpha]_{\mathrm{D}}^{24} 36.3\left(\mathrm{c}=0.49, \mathrm{CHCl}_{3}\right)$

TLC: $\quad R_{f} 0.15$ (hexanes/EtOAc, 4:1) [UV (254 nm)]

SFC: $\quad(2 R, 3 S)-\mathbf{6 f a}, t_{\mathrm{R}} 19.9 \min (93.2 \%) ;(R, R) /(S, S)-\mathbf{6 f a}, t_{\mathrm{R}} 14.0 \min (2.1 \%) ;(2 S, 3 R)-\mathbf{6 f a}$, $t_{\mathrm{R}} 15.2 \min (3.2 \%) ;(R, R) /(S, S)-6 \mathbf{f a}, t_{\mathrm{R}} 16.3 \min (1.4 \%)$ (Chiralcel OD, 150 bar, 40 ${ }^{\circ} \mathrm{C}, 6 \% \mathrm{MeOH}$ in $\left.\mathrm{CO}_{2}, 2.2 \mathrm{~mL} / \mathrm{min}\right)$

Analysis: $\mathrm{C}_{17} \mathrm{H}_{17} \mathrm{NO}_{2}(267.32)$

Calcd: $\quad$ C, 76.38; H, 6.41; N, 5.24\%

Found: C, 76.66; H, 6.39; N, 5.29\% 
Preparation of (2R,3S)-3-Hydroxy-2-methyl-3-(methylphenyl)-2-phenylpropanenitrile (6ga) (Table 3, entry 5)
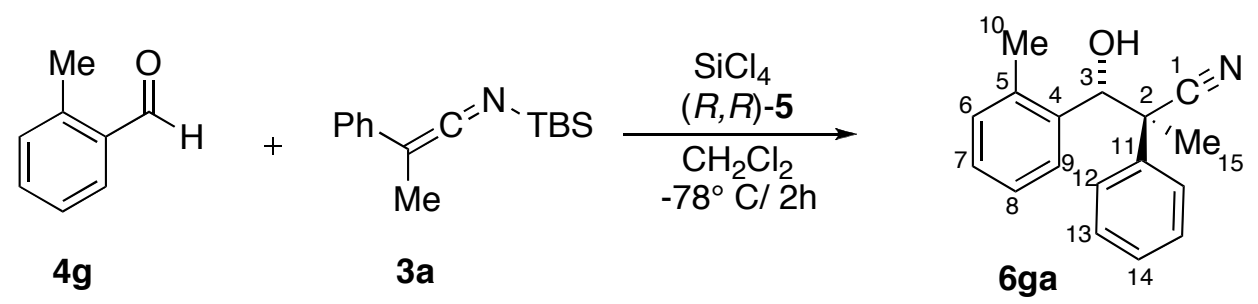

Following General Procedure 2, $120.6 \mathrm{mg}$ of 2-tolualdehyde (1.0 mmol, 1.0 equiv) was combined with $42 \mathrm{mg}$ of $(R, R)-5$ (0.05 mmol, 0.05 equiv), $126 \mu \mathrm{L}$ of $\mathrm{SiCl}_{4}(1.1 \mathrm{mmol}, 1.1$ equiv) and $0.86 \mathrm{~mL}$ of a $1.4 \mathrm{M}$ solution of silyl ketene imine 3a (1.2 mmol, 1.2 equiv, $1.4 \mathrm{M}_{\text {in }} \mathrm{CH}_{2} \mathrm{Cl}_{2}$ ) to yield after column chromatography $\left(\mathrm{SiO}_{2}(30 \mathrm{~g}) 3 \mathrm{~cm}\right.$ diam. hexanes/EtOAc 4:1 ) $237 \mathrm{mg}$ (94\%) of 6ga as a white solid, which was crystallized from hot EtOAc (1 mL) and hexanes (1 $\mathrm{mL})$ to afford $213 \mathrm{mg}(84 \%)$ of $\mathbf{6 g a}$ as white plates.

Data for 6ga:

mp: $\quad 154-155^{\circ} \mathrm{C}$

${ }^{1} \mathrm{H}$ NMR: $\quad\left(500 \mathrm{MHz}, \mathrm{CDCl}_{3}\right)$

7.65 (dd, $J=7.5,1.9,1 \mathrm{H}, \mathrm{HC}(8)), 7.47-7.43$ (m, $2 \mathrm{H}, \mathrm{HC}(13))$, 7.41-7.34 (m, $3 \mathrm{H}$, HC(12, 9)), 7.29-7.22 (m, 2 H, HC(14, 8)), 7.12-7.09 (m, 1 H, HC(7)), 5.22 (s, 1 H, $\mathrm{HC}(3)$ ), 2.35 (br. s, $1 \mathrm{H}, \mathrm{OH}), 2.09$ (s, $3 \mathrm{H}, \mathrm{H}_{3} \mathrm{C}(10)$ ), 1.65 (s, $3 \mathrm{H}, \mathrm{H}_{3} \mathrm{C}(15)$ )

${ }^{13} \mathrm{C} \mathrm{NMR:} \quad\left(125 \mathrm{MHz}, \mathrm{CDCl}_{3}\right)$

$137.6(\mathrm{C}(4,11,9)), 136.3(\mathrm{C}(4,11,9)), 136.1 \quad(\mathrm{C}(4,11,9)), \quad 130.3(\mathrm{C}(7)), 128.8$ (C(12)), $128.5(\mathrm{C}(8)), 128.3$ (C(9)), 127.1 (C(8)), 126.8 (C(13)), 126.2 (C(14)), 122.1 (C(1)), 74.3 (C(3)), 45.0 (C(15)), 19.5 (C(10))

IR: $(\mathrm{KBr}$ pellet) 3416 (s), 3061 (m), 3018 (m), 2996 (m), 2939 (m), 2245 (s), 1977 (w), 1956 (w), 1921 (w), 1895 (w), 1876 (w), 1840 (w), 1809 (w), 1600 (m), 1499 (s), 1464 (s), 1447 (s), 1387 (s), 1043 (s), 1027 (s), 782 (s), 735 (s), 710 (s), 695 (s), 615 (m)

MS: (ESI) 132 (16), 207 (13), 234 (48), 274 (48), $275\left(\mathrm{M}^{+}+\mathrm{Na}, 16\right)$

$\underline{\text { HRMS }}$ Calcd for $\mathrm{C}_{17} \mathrm{H}_{17} \mathrm{NONa}$ : 274.1218, found: 274.1208 
Opt. Rot.: $[\alpha]_{\mathrm{D}}^{24} 44.6\left(\mathrm{c}=0.49, \mathrm{CHCl}_{3}\right)$

TLC: $\mathrm{R}_{f} 0.25$ (hexanes/EtOAc, 4:1) [UV (254 nm)]

HPLC: $\quad(2 R, 3 S)-6$ ga, $t_{\mathrm{R}} 32.1 \mathrm{~min}(98.4 \%) ;(R, R) /(S, S)-6 \mathbf{g a}, t_{\mathrm{R}} 28.7 \mathrm{~min}(0.7 \%) ;(2 S, 3 R)-6 \mathbf{g a}$, $t_{\mathrm{R}} 38.5(0.8 \%)$ (Chiralpak AD-RH, gradient from $30 \% \mathrm{MeCN}$ in $\mathrm{H}_{2} \mathrm{O}(0.5 \% \mathrm{AcOH})$ to $50 \% \mathrm{MeCN}$ over $30 \mathrm{~min}, 0.5 \mathrm{~mL} / \mathrm{min}$ )

Analysis: $\mathrm{C}_{17} \mathrm{H}_{17} \mathrm{NO}(251.32)$

Calcd: $\quad$ C, 81.24; H, 6.82; N, $5.57 \%$

Found: $\quad \mathrm{C}, 80.94 ; \quad \mathrm{H}, 6.83 ; \quad \mathrm{N}, 5.81 \%$

Preparation of (2R,3S)-3-Hydroxy-2-methyl-3-(1-naphthyl)-2-phenylpropanenitrile (6ha) (Table 3, entry 6)<smiles>O=C(I)c1cccc2ccccc12</smiles>

$4 h$<smiles>CC(=C=N[As]c1ccccc1)c1ccccc1</smiles>

$3 a$

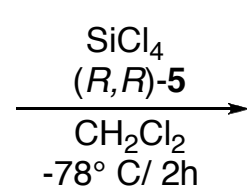

$-78^{\circ} \mathrm{C} / 2 \mathrm{~h}$

Following General Procedure 2, $156 \mathrm{mg}$ of 1-naphthaldehyde (1.0 mmol, 1.0 equiv) was combined with $42 \mathrm{mg}$ of $(R, R)-5$ (0.05 mmol, 0.05 equiv), $126 \mu \mathrm{L}$ of $\mathrm{SiCl}_{4}(1.1 \mathrm{mmol}, 1.1$ equiv) and $0.86 \mathrm{~mL}$ of a $1.4 \mathrm{M}$ solution of silyl ketene imine 3a (1.2 mmol, 1.2 equiv, $1.4 \mathrm{M}_{\text {in }} \mathrm{CH}_{2} \mathrm{Cl}_{2}$ ) to yield after column chromatography $\left(\mathrm{SiO}_{2}(25 \mathrm{~g}) 3 \mathrm{~cm}\right.$ diam. hexanes/EtOAc 4:1 ) $270 \mathrm{mg}$ $(94 \%)$ of 6 ha a a yellow foam, which was recrystallized twice from hot toluene $(0.2 \mathrm{~mL})$ to yield $218 \mathrm{mg}(76 \%)$ of 6 ha as white plates.

Data for 6ha:

mp: $87-89^{\circ} \mathrm{C}$

${ }^{1} \mathrm{H}$ NMR: $\quad\left(500 \mathrm{MHz}, \mathrm{CDCl}_{3}\right)$

8.04-8.00 (m, $1 \mathrm{H}, \mathrm{HC}(17))$, 7.95-7.91 (m, $1 \mathrm{H}, \mathrm{HC}(11))$, 7.90-7.87 (m, $2 \mathrm{H}$, $\mathrm{HC}(6,9))$, 7.63-7.57 (m, 3 H, HC(16,7,8)), 7.50-7.45 (m, 2 H, HC(12,13)), 7.457.40 (m,2 H, HC(15)), 7.40-7.35 (m,1 H, HC(7,8)), 5.86 (s, 1H, HC(3)), 2.31 (br. s, $1 \mathrm{H}, \mathrm{OH}), 1.55$ (s, 3H, $\left.\mathrm{H}_{3} \mathrm{C}(18)\right)$

${ }^{13} \mathrm{C}$ NMR: $\quad\left(125 \mathrm{MHz}, \mathrm{CDCl}_{3}\right)$

$138.2(\mathrm{C}(14)), 134.2(\mathrm{C}(5,10,4)), 133.3(\mathrm{C}(5,10,4)), 131.4 \quad(\mathrm{C}(5,10,4)), 129.3$ 
(C(6,9)), $129.0(\mathrm{C}(6,7,8,9,11,12,13), 128.9$ (C(15)), $128.4(\mathrm{C}(7,8)), 126.6(\mathrm{C}(16))$,

$126.2 \quad(\mathrm{C}(12,13)), \quad 125.6 \quad(\mathrm{C}(17)), \quad 125.4 \quad(\mathrm{C}(6,7,8,9,11,12,13)), \quad 125.4$ (C(6,7,8,9,11,12,13)), $122.4(\mathrm{C}(6,7,8,9,11,12,13)), 121.7(\mathrm{C}(1)), 73.7$ (C(3)), 50.9 $(\mathrm{C}(2)), 23.1(\mathrm{C}(18))$

IR: $(\mathrm{KBr}$ pellet)

3445 (m), 3059 (w), 3002 (w), 2984 (w), 2939 (w), 2917 (w), 2249 (w), 1596 (w), 1512 (2), 1495 (w), 1454 (w), 1444 (w), 1390 (w), 1376 (w), 1345 (w), 1305 (w), 1257 (w), 1213 (w), $1166(\mathrm{w}), 1073$ (w), 1050 (m), 1025 (m), 802 (m), 783 (s), 698 (m)

MS: (ESI)

243 (35), 270 (100), 271 (40), 305 (39), $310\left(\mathrm{M}^{+}+\mathrm{Na}, 33\right)$

$\underline{\text { HRMS }}$ Calcd for $\mathrm{C}_{20} \mathrm{H}_{17} \mathrm{NONa}$ : 310.1208 , found: 310.1206

Opt. Rot.: $[\alpha]_{\mathrm{D}}^{24} 15.2\left(\mathrm{c}=0.51, \mathrm{CHCl}_{3}\right)$

TLC: $\quad \mathrm{R}_{f} 0.26$ (hexanes/EtOAc, 4:1) [UV (254 nm)]

SFC: $\quad(2 R, 3 S)-6 \mathbf{h a}, t_{\mathrm{R}} 11.3 \min (98.3 \%) ;(R, R) /(S, S)-6 \mathbf{h a}, t_{\mathrm{R}} 13.3 \min (0.4 \%)$; $(2 S, 3 R)-6 \mathbf{h a}$, $t_{\mathrm{R}} 16.8 \mathrm{~min}(1.2 \%)$ (Chiralpak AS, $125 \mathrm{bar}, 40^{\circ} \mathrm{C}, 8 \% \mathrm{MeOH}$ in $\mathrm{CO}_{2} 3.0 \mathrm{~mL} / \mathrm{min}$ )

Analysis: $\quad \mathrm{C}_{20} \mathrm{H}_{17} \mathrm{NO}(287.35)$

Calcd: $\quad$ C, 83.59; H, 5.96; N, $4.87 \%$

Found: $\quad \mathrm{C}, 83.31 ; \quad \mathrm{H}, 5.88 ; \quad \mathrm{N}, 4.93 \%$

Preparation of (2R,3R)-3-(2-Furyl)-3-hydroxy-2-methyl-2-phenylpropanenitrile (6ia) (Table 3, entry 7)<smiles>O=Cc1ccco1</smiles>

$4 \mathbf{i}$<smiles></smiles>

$3 \mathbf{a}$

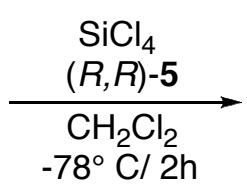

6ia

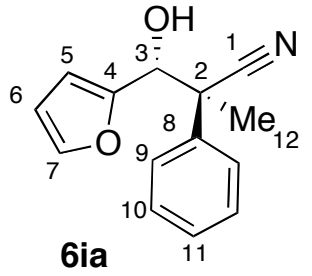

Following General Procedure 2, $96.0 \mathrm{mg}$ of 2-furaldehyde (1.0 mmol, 1.0 equiv) was combined with $42 \mathrm{mg}$ of $(R, R)-5\left(0.05 \mathrm{mmol}, 0.05\right.$ equiv), $126 \mu \mathrm{L}$ of $\mathrm{SiCl}_{4}(1.1 \mathrm{mmol}, 1.1$ equiv) and $0.86 \mathrm{~mL}$ of a $1.4 \mathrm{M}$ solution of silyl ketene imine 3a (1.2 mmol, 1.2 equiv, $1.4 \mathrm{M}$ in $\mathrm{CH}_{2} \mathrm{Cl}_{2}$ ) to yield after column chromatography $\left(\mathrm{SiO}_{2}(29 \mathrm{~g}) 3 \mathrm{~cm}\right.$ diam hexanes/EtOAc 4:1 ) $219 \mathrm{mg}$ 
$(96 \%)$ of 6 ia as a white solid, which was sublimed under high vacuum $\left(75^{\circ} \mathrm{C}\right)$ to yield $209 \mathrm{mg}$ $(92 \%)$ of $6 \mathbf{i a}$ as white needles.

Data for 6ia:

mp: $79-81^{\circ} \mathrm{C}$

${ }^{1} \mathrm{H}$ NMR: $\quad\left(500 \mathrm{MHz}, \mathrm{CDCl}_{3}\right)$

7.47-7.44 (m, 2 H, HC(9)), 7.41-7.32 (m, 2 H, HC(7,10,11)), 6.41 (d, J = 3.4, 1 H, HC(5)), 6.37 (dd, $J=1.8,3.3, \mathrm{HC}(6)), 4.89$ (s, $1 \mathrm{H}, \mathrm{HC}(3)$ ), 2.74 (br s, $1 \mathrm{H}, \mathrm{OH}$ ), $1.64\left(\mathrm{~s}, 3 \mathrm{H}, \mathrm{H}_{3} \mathrm{C}(12)\right)$

${ }^{13} \mathrm{C} \mathrm{NMR:} \quad\left(125 \mathrm{MHz}, \mathrm{CDCl}_{3}\right)$

$151.5(\mathrm{C}(4)), 142.3$ (C(7)), 137.2 (C(8)), 128.7 (C(10)), 128.3 (C(11)), 126.3 (C(9)), 121.5 (C(1)), 110.5 (C(6)), 108.7 (C(5)), 73.8 (C(3)), 48.9 (C(2)), 22.2 (C(12))

IR: $(\mathrm{KBr}$ pellet $)$

3400 (s), 3147 (w), 3123 (w), 3058 (w), 3026 (w), 2994 (w), 2869 (m), 2256 (m), 1615 (w), 1598 (m), 1584 (w), 1499 (s), 1448 (s), 1382 (s), 1296 (s), 1274 (m), 1188 (m), 1155 (m), 1088 (s), 1077 (s), 1072 (s), 1048 (s), 1009 (s), 741 (s), 694 (s)

MS: (ESI)

210 (100), $211(13), 250\left(\mathrm{M}^{+}+\mathrm{Na}, 62\right), 360$ (2)

$\underline{\text { HRMS }}$ Calcd for $\mathrm{C}_{14} \mathrm{H}_{13} \mathrm{NO}_{2} \mathrm{Na}: 250.0844$, found: 250.0845

Opt. Rot.: $[\alpha]_{\mathrm{D}}{ }^{24} 35.3\left(\mathrm{c}=0.50, \mathrm{CHCl}_{3}\right)$

TLC: $\quad \mathrm{R}_{f} 0.14$ (hexanes/EtOAc, 4:1) [UV (254 nm)]

SFC: $\quad(2 R, 3 S)$-6ia, $t_{\mathrm{R}} 3.2 \min (93.9 \%) ;(R, R) /(S, S)$-6ia, $t_{\mathrm{R}} 2.8 \min (1.0 \%) ;(2 S, 3 R)-6 \mathbf{i a}, t_{\mathrm{R}}$ $3.5 \mathrm{~min},(5.08 \%)$ (Chiralpak AD, $120 \mathrm{bar}, 40{ }^{\circ} \mathrm{C}, 9 \% \mathrm{MeOH}$ in $\left.\mathrm{CO}_{2}, 3.2 \mathrm{~mL} / \mathrm{min}\right)$

Analysis: $\mathrm{C}_{14} \mathrm{H}_{13} \mathrm{NO}_{2}(227.25)$

Calcd: $\quad$ C, 73.99; H, 5.77; N, 6.16\%

Found: C, 73.80; H, 5.72; N, 6.30\% 


\section{References}

(1) Hoye, T. R.; Eklov, B. M.; Voloshin, M. Org. Lett. 2004, 6, 2567-2570

(2) Watt, D. S. Tetrahedron Lett. 1974, 15, 707-710.

(3) Freerksen, R. W.; Silikson, S. J.; Wroble, R. R.; Kyler, K. S.; Watt, D. S. J. Org. Chem. 1983, 48, 4087-4096.

(4) Blanchard, E. P., Jr.; Cairncross, A. J. Am. Chem. Soc. 1966, 88, 487-495.

(5) Mermerian, A. H.; Fu, G. Angew. Chem., Int. Ed. 2005, 44, 949-952.

(6) Watt, D. S. Syn. Commun. 1974, 4, 127-132.

(7) The crude material was concentrated at $5 \mathrm{mmHg}$ due to volatility of the product.

(8) Absolute and relative configuration of the minor diastereomer is based on ${ }^{1} \mathrm{H}$ NMR chemical shift correlations to the other nitrile products and has not been unambiguously assigned.

(9) Compound $(2 R, 3 S)$-6bj was obtained with $\mathrm{dr}>99: 1$ by NMR integration. Compound $(2 S, 3 S)-\mathbf{6 b j}$ was obtained as a mixture of diastereomers with a ratio of 87:13 by NMR integration. 
exp1 s2pul

\begin{tabular}{|c|c|c|c|}
\hline \multicolumn{2}{|c|}{ SAMPLE } & \multicolumn{2}{|c|}{ DEC. \& VT } \\
\hline date & $20 \quad 2007$ & dfrq & 499.699 \\
\hline solve: & Benzene & $d n$ & H1 \\
\hline \multicolumn{2}{|c|}{ file /export/home/ } & dpwr & 20 \\
\hline \multirow{2}{*}{\multicolumn{2}{|c|}{$\begin{array}{l}\text { user1d/data/u500/b } \\
\text { urk/MTB-II-23.fid }\end{array}$}} & dof & 0 \\
\hline & & $\mathrm{dm}$ & $\mathrm{nnn}$ \\
\hline \multicolumn{2}{|c|}{ ACQUISITION } & $\mathrm{dmm}$ & c \\
\hline sfrq & 499.699 & $\operatorname{dmf}$ & 200 \\
\hline tn & H1 & dseq & \\
\hline at & 4.665 & dres & 1.0 \\
\hline np & 65536 & homo & $\mathrm{n}$ \\
\hline sw & 7024.9 & \\
\hline $\mathrm{fb}$ & 4000 & \multicolumn{2}{|c|}{ DEC2 } \\
\hline bs & 4 & \multicolumn{2}{|l|}{$\operatorname{dn} 2$} \\
\hline tpwr & 63 & \multicolumn{2}{|l|}{ dpwr2 } \\
\hline pw & 6.5 & \multicolumn{2}{|l|}{$\operatorname{dof} 2$} \\
\hline d1 & 10.000 & \multicolumn{2}{|l|}{$\mathrm{dm} 2$} \\
\hline tof & 2.0 & \multicolumn{2}{|l|}{$\mathrm{dmm} 2$} \\
\hline nt & 4 & \multicolumn{2}{|l|}{$\operatorname{dmf} 2$} \\
\hline ct & 4 & \multicolumn{2}{|l|}{ dseq2 } \\
\hline alock & $\mathrm{n}$ & \multicolumn{2}{|l|}{ dres2 } \\
\hline gain & not used & homo2 & $\mathrm{n}$ \\
\hline \multicolumn{2}{|c|}{ FLAGS } & \multicolumn{2}{|c|}{ PROCESSING } \\
\hline il & $\mathrm{n}$ & $1 \mathrm{~b}$ & 0.30 \\
\hline in & $\mathrm{n}$ & wtfile & \\
\hline $\mathrm{dp}$ & $\mathrm{y}$ & proc & $\mathrm{ft}$ \\
\hline hs & $\mathrm{nn}$ & fn & not used \\
\hline \multicolumn{2}{|c|}{ DISPLAY } & math & $\mathrm{f}$ \\
\hline sp & -250.0 & & \\
\hline wp & 5496.6 & werr & \\
\hline vs & 18 & wexp & \\
\hline sc & 0 & wbs & \\
\hline wc & 250 & wnt & \\
\hline hzmm & 21.99 & & \\
\hline is & 4.20 & & \\
\hline$r f l$ & 4571.7 & & \\
\hline$r f p$ & 3572.8 & & \\
\hline th & 7 & & \\
\hline ins & 3.000 & & \\
\hline$a i$ & & & \\
\hline
\end{tabular}<smiles>COc1ccc(C(C)=C=N[AsH2+][SbH2])cc1</smiles>

$3 c$ 


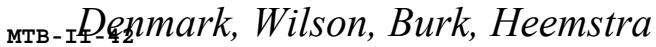

exp1 s2pul

SAMPLE DEC. \& VT

date Aug 162007 dfrq $\quad 499.699$

solvent Benzene dn H1

$\begin{array}{lr}\text { file /export/home/ dpwr } & 20 \\ \text { userld/data/u500/b dof } & 0\end{array}$

urk/MTB-II-42.fid dm

ACOUISITION $\mathrm{nnn}$

$\begin{array}{llll}\text { sfrq } & 499.699 & \mathrm{dmf} & 200\end{array}$

$\begin{array}{rrll}\text { tn } & \text { H1 } & \text { dseq } & \\ \text { at } & 4.665 & \text { dres } & 1.0\end{array}$

np $\quad 65536$ homo $\quad 1.0$

$\begin{array}{lrll}\text { Sw } & 7024.9 & & \text { DEC2 } \\ \mathrm{fb} & 4000 & \text { dfrq2 } & \end{array}$

$\begin{array}{lrl}\text { bs } & 4 & \text { dn2 } \\ \text { tpwr } & 63 & \text { dpwr2 }\end{array}$

$\begin{array}{lrll}\text { tpwr } & 63 & \text { dpwr2 } & 1 \\ \text { pw } & 6.5 & \text { dof2 } & 0\end{array}$

$\begin{array}{lrll}\text { pw } & 6.5 & \text { dof2 } & 0 \\ \text { d1 } & 10.000 & \text { dm2 } & n \\ \text { tof } & 2.0 & \text { dmm2 } & c\end{array}$

$\begin{array}{lrlr}\text { tof } & 2.0 & \text { dmm2 } & c \\ \text { nt } & 4 & \text { dmf2 } & 200\end{array}$

$\begin{array}{llll}\text { nt } & 4 & \text { dmf2 } & 200 \\ \text { ct } & 4 & \text { dseq2 } & \end{array}$

$\begin{array}{lrrr}\text { alock } & \mathrm{n} & \text { dres2 } & 1.0 \\ \text { gain } & \text { not used } & \text { homo2 } & \mathrm{n}\end{array}$

gain not used homo2
FLAGS

$\begin{array}{lll}\text { il } & \mathrm{n} \text { lb } & 0.30\end{array}$

$\begin{array}{lll}\text { in } & n & \text { wtfile } \\ \text { dp } & y & \text { proc }\end{array}$

hs nn fn not used

DISPLAY math

$\begin{aligned}-249.9 & \\ 5496.6 & \text { werr }\end{aligned}$

31 wexp

0 wbs

250 wnt

wc 250

$\begin{array}{ll}\text { hzmm } & 21.99 \\ \text { is } & 33.57\end{array}$

is $\quad 33.57$

rfp $\quad 3572.8$

$\begin{array}{lr}\text { th } & 7 \\ \text { ins } & 3.000\end{array}$

$\begin{array}{ll}\text { ins } & 3.000 \\ \text { ai ph }\end{array}$

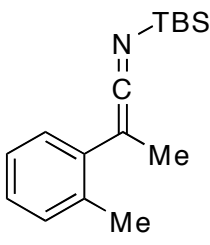

$3 d$

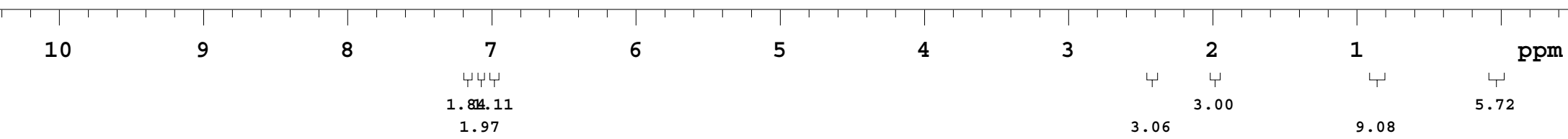




\section{Denmark, Wilson, Burk, Heemstra}

1HNMR- twW-VII- 025 -crude- $d 1=10$ s

exp1 s2pul

SAMPLE DEC. \& VT

date Aug 232007 dfrq $\quad 500.070$

solvent Benzene dn H1

file /export/home/ dpwr 18

user1d/data/ui500n dof

b/wilsont/1HNMR-tw $\sim d m$

w-VII-025-crude-d1 dmm

$=10$ s.fid $\mathrm{dmf}$

ACQUISITION dseq

sfrq $\quad 500.070$ dres

tn H1 homo

at $4.096 \quad$ DEC2

np 65536 dfrq2

$8000.0 \mathrm{dn} 2$

4000 dpwr2

$\begin{array}{lrl}\text { fb } & 4000 & \text { dpwr2 } \\ \text { bs } & 16 & \text { dof2 } \\ \text { tpwr } & 55 & \text { dm2 }\end{array}$

$\begin{array}{lrl}\text { tpwr } & 55 & \mathrm{dm} 2 \\ \text { pw } & 7.7 & \mathrm{dmm} 2\end{array}$

$\begin{array}{lrl}\text { pw } & 7.7 & \text { dmm2 } \\ \text { d1 } & 10.000 & \text { dmf2 } \\ \text { tof } & 0 & \text { dseq2 }\end{array}$

$\begin{array}{lllr}\text { nt } & 16 & \text { dres2 } & 1.0 \\ \text { ct } & 16 & \text { homo2 } & n\end{array}$

alock $n$ PROCESSING

gain not used lb 0.30

FLAGS $n$ wtfile

$\begin{array}{rrrr}\text { in } & \text { n } & \text { fn } & \text { not used } \\ \text { dp } & y & \text { math } & f \\ \text { hs } & \text { nn } & & \end{array}$

hs DISPLAY

DISPLAY werr

-250.1 wexp

5500.7 wbs

$\begin{array}{lrl}\text { wp } & 5500.7 & \text { wbs } \\ \text { vs } & 22 & \text { wnt }\end{array}$

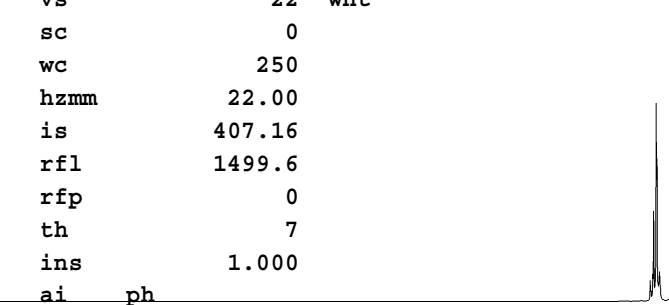

$S 40 \mathrm{Me}_{\mathrm{Me}}^{\mathrm{M}}$

$3 g$

ai ph Nh

10

9

8

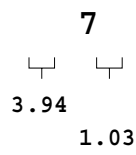

6

5

4

3

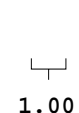

2

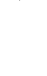

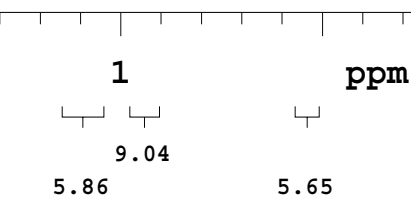


Denmark, Wilson, Burk, Heemstra 1HNMR- tww-VI-099-d1-d110s

exp1 s2pul

SAMPLE DEC. \& VT

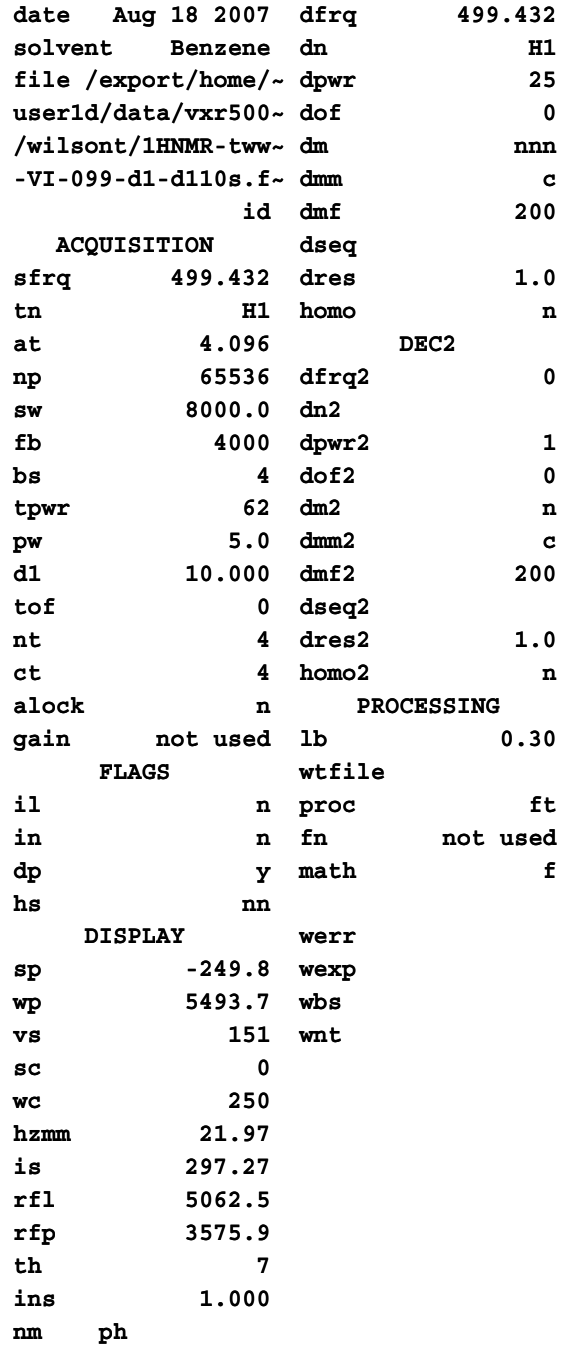

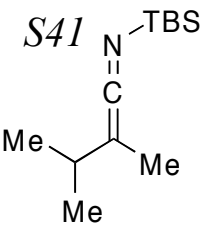

3j

ACOUISITION dseg

alock $n$ PROCESSING

a

